\title{
Immune response to intravenous immunoglobulin in patients with Kawasaki disease and MIS-C
}

\author{
Yanfang P. Zhu, ${ }^{1}$ Isaac Shamie, ${ }^{2}$ Jamie C. Lee, ${ }^{1,2}$ Cameron J. Nowell, ${ }^{3}$ Weiqi Peng, ${ }^{1,4}$ Shiela Angulo, ${ }^{1}$ Linh N.N. Le, ${ }^{1,2}$ Yushan Liu, ${ }^{1,5}$ \\ Huilai Miao, ${ }^{1}$ Hainan Xiong, ${ }^{1}$ Cathleen J. Pena, ${ }^{1}$ Elizabeth Moreno, ${ }^{1}$ Eric Griffis, ${ }^{6}$ Stephanie G. Labou, ${ }^{7}$ Alessandra Franco, ${ }^{1}$ \\ Lori Broderick,, ${ }^{1,8}$ Hal M. Hoffman,, ${ }^{1,8}$ Chisato Shimizu, ${ }^{1}$ Nathan E. Lewis, ${ }^{1,2}$ John T. Kanegaye, ${ }^{1,8}$ Adriana H. Tremoulet, ${ }^{1,8}$ \\ Jane C. Burns, ${ }^{1,8}$ Ben A. Croker, ${ }^{1}$ and the Pediatric Emergency Medicine Kawasaki Disease Research Group Consortium ${ }^{8,9}$ \\ 'Department of Pediatrics and ${ }^{2}$ Department of Bioengineering, UCSD, La Jolla, California, USA. ${ }^{3}$ Monash Institute of Pharmaceutical Sciences, Parkville, Victoria, Australia. ${ }^{4}$ Department of Mathematics, \\ ${ }^{5}$ Department of Computer Science and Engineering, ${ }^{6}$ Nikon Imaging Center, and ${ }^{7}$ UCSD Library, UCSD, California, USA. ${ }^{8}$ Rady Children's Hospital San Diego, San Diego, California, USA. ${ }^{9}$ The Pediatric \\ Emergency Medicine Kawasaki Disease Research Group Consortium is detailed in Supplemental Acknowledgments.
}

\begin{abstract}
BACKGROUND. Multisystem inflammatory syndrome in children (MIS-C) is a rare but potentially severe illness that follows exposure to severe acute respiratory syndrome coronavirus 2 (SARS-CoV-2). Kawasaki disease (KD) shares several clinical features with MIS-C, which prompted the use of intravenous immunoglobulin (IVIC), a mainstay therapy for KD. Both diseases share a robust activation of the innate immune system, including the IL-1 signaling pathway, and IL-1 blockade has been used for the treatment of both MIS-C and KD. The mechanism of action of IVIC in these 2 diseases and the cellular source of IL-1 $\beta$ have not been defined.
\end{abstract}

\begin{abstract}
METHODS. The effects of IVIG on peripheral blood leukocyte populations from patients with MIS-C and KD were examined
\end{abstract} using flow cytometry and mass cytometry (CуTOF) and live-cell imaging.

RESULTS. Circulating neutrophils were highly activated in patients with KD and MIS-C and were a major source of IL-1ק. Following IVIG treatment, activated IL-1 $\beta^{+}$neutrophils were reduced in the circulation. In vitro, IVIG was a potent activator of neutrophil cell death via PI3K and NADPH oxidase, but independently of caspase activation.

CONCLUSIONS. Activated neutrophils expressing IL-1 $\beta$ can be targeted by IVIG, supporting its use in both KD and MIS-C to ameliorate inflammation.

FUNDING. Patient Centered Outcomes Research Institute; NIH; American Asthma Foundation; American Heart Association; Novo Nordisk Foundation; NIGMS; American Academy of Allergy, Asthma and Immunology Foundation.

\section{Introduction}

Multisystem inflammatory syndrome in children (MIS-C) may occur following exposure to severe acute respiratory syndrome coronavirus 2 (SARS-CoV-2) and is characterized by the acute onset of fever that may be variably associated with rash, conjunctival injection, mucocutaneous erythema, coagulopathy, cardiovascular complications, including shock and coronary artery dilation, and gastrointestinal symptoms, including abdominal pain, diarrhea, and vomiting (1-7). Kawasaki disease (KD) can cause myocarditis and a vasculitis of the coronary arteries, leading to aneurysm formation in untreated children with $\operatorname{KD}(8,9)$. The major sequelae of aneurysms include thrombosis, late coronary artery stenosis, myocardial ischemia, myocardial infarction, and death (10-12). Cardiac abnormalities in MIS-C tend to resolve quickly with treatment and include conduction abnormalities, coronary

Conflict of interest: The authors have declared that no conflict of interest exists. Copyright: @ 2021, American Society for Clinical Investigation.

Submitted: December 21, 2020; Accepted: August 24, 2021; Published: October 15, 2021. Reference information: J Clin Invest. 2021;131(20):e147076.

https://doi.org/10.1172/JCl147076. artery dilatation, aneurysms, and ventricular dysfunction. However, the long-term sequelae are unknown (13). In severe cases, vasodilatory or cardiogenic shock has been reported requiring inotropic support and, in rare cases, extracorporeal membrane oxygenation (14). MIS-C differs from KD in that it is more common in older children, and gastrointestinal symptoms are frequently reported. Children of Hispanic and African-American descent are at higher risk for MIS-C, whereas children of Asian descent have a higher risk of KD (15).

Clinical trials in the 1980s established that a high dose of intravenous immunoglobulin (IVIG) administered within the first 10 days after fever onset reduced the incidence of coronary artery aneurysms in KD (16). IVIG is now standard of care for KD and can alleviate symptoms and stabilize patients within hours of administration (2). The clinical presentation of MIS-C suggests that the two diseases might share common immune drivers, and IVIG was adopted as first-line therapy to treat MIS-C (17). In contrast to what occurs with $\mathrm{KD}$, however, additional antiinflammatory therapy with corticosteroids, TNF antagonists, and IL-1 antagonists is often needed to suppress inflammation. Recent studies suggested an active role of blood neutrophils in mediating hyperinflamma- 

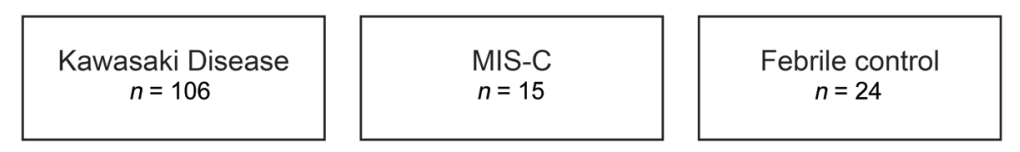

Figure 1. Patient enrollment and follow-up. Patients with $\mathrm{KD}$ and MIS-C were studied prior to treatment, early after IVIG administration ( 9 to 30 hours), or in a subacute phase 2 to 6 weeks after IVIC treatment. Blood samples were analyzed by CBC, CyTOF, flow cytometry, and live-cell imaging.
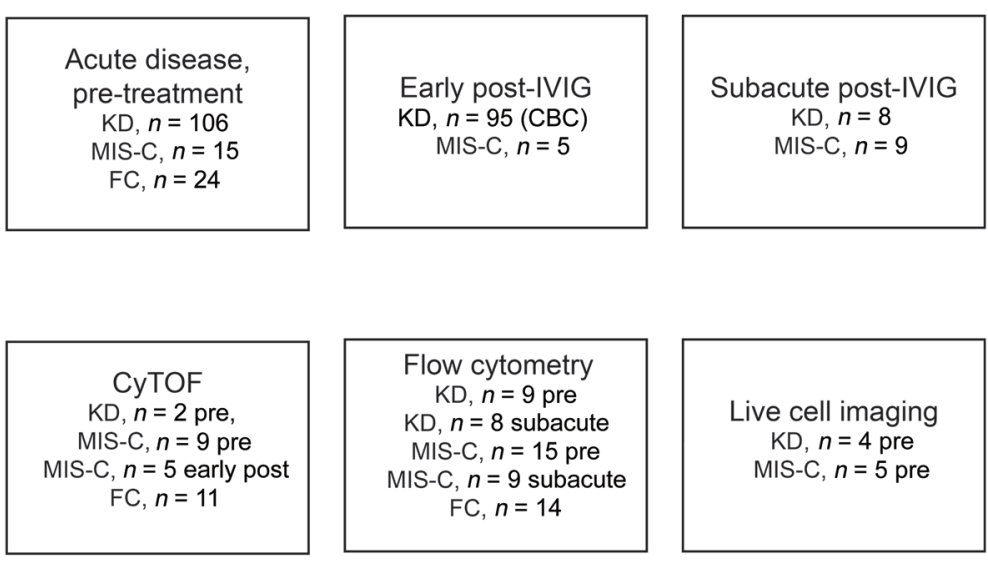

tion in patients infected with SARS-CoV-2 $(18,19)$. Hyperinflammation is also seen in MIS-C, although the mechanism of the hyperinflammation remains unclear. Immunophenotyping using flow cytometry and mass cytometry (CyTOF) revealed reductions of several subsets of monocytes and lymphocytes in the blood of MIS-C patients with active disease and ablation of activated neutrophils following treatment $(13,20)$. Gene-expression studies of peripheral blood leukocytes indicate that IL-1 family signaling intermediates are elevated in patients with $\mathrm{KD}$ as well as in patients with MIS-C $(20,21)$. A role for IL-1 signaling is also supported by genetic studies in murine models of $\mathrm{KD}$ featuring aortic dilation and aneurysm formation $(22,23)$. The frequent need for adjunctive therapy in patients with MIS-C highlights the need for a better understanding of the disease process and the mechanism of action of frontline therapies, including IVIG. In this study, we examine the effects of IVIG on peripheral blood leukocyte populations and compare the expression of IL- $1 \beta$ in leukocytes, particularly in neutrophils, in patients with KD and MIS-C.

\section{Results}

Neutrophilia in MIS-C and KD. To understand the unique features of MIS-C and KD, we obtained blood samples and confirmed a diagnosis of KD or MIS-C based on a combination of serology for SARS-CoV-2-specific antibodies and $\mathrm{T}$ cell reactivity to SARSCoV-2 epitopes (Figure 1 and ref. 24). MIS-C patients were significantly older than patients with $\mathrm{KD}$ and had higher $\mathrm{C}$-reactive protein levels than febrile controls (FCs) (Table 1). To understand how peripheral blood leukocytes contribute to systemic inflammation in KD and MIS-C, we investigated the composition of the peripheral blood population by flow cytometry immediately after phlebotomy (Figure 2A and Table 2). In our small series, lymphopenia was common among patients with KD and MIS-C and FCs
(Table 1, Figure 2B, and Supplemental Figure 1A; supplemental material available online with this article; https://doi.org/10.1172/ JCI147076DS1). No significant difference was noted in the monocyte population, but an increase in eosinophils and neutrophils was noted in KD patients compared with FCs (Figure 2B and Supplemental Figure 1A). The absolute neutrophil count in the KD cohort was 2.6-fold higher than in FCs (Figure 2B).

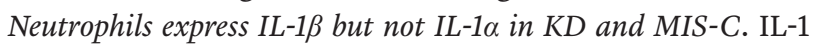
receptor signaling has been implicated in the pathogenesis of KD and MIS-C, with IL-1 antagonists currently in use for both patient populations $(2,15,20-22,25-27)$. However, the key cell types releasing IL- $1 \alpha$ and/or IL-1 $\beta$ have not been established. To investigate circulating leukocytes expressing IL-1 $\beta$ in KD and MIS-C, we performed intracellular flow cytometry using AF647-conjugated canakinumab, a human IL-1 $\beta$-specific monoclonal antibody (Figure $2 \mathrm{C}$ and ref. 28). We observed increased numbers of IL-1 $\beta^{+}$cells in KD patients compared with FCs (Figure 2D). In patients with KD and MIS-C, the proportion of IL- $1 \beta^{+}$cells was $50 \%$ to $80 \%$ of blood leukocytes (Supplemental Figure 1B). The median counts of IL-1 $\beta^{+}$ cells were about 5-fold higher in the KD cohort and about 2-fold higher in the MIS-C cohort than in the FC cohort (Figure 2D). IL- $1 \beta^{+}$leukocytes were predominately neutrophils ( $\left.80 \%\right)$ and to a lesser extent eosinophils and monocytes ( $10 \%$; Figure $2 \mathrm{E})$. Analysis of mRNA from neutrophils of MIS-C patients by quantitative reverse-transcriptase PCR (RT-PCR) confirmed the expression of IL1B mRNA, but not IL1A mRNA (Figure 2F). Blood samples from FCs also demonstrated IL-1 $\beta$ expression in neutrophils as well as eosinophils, monocytes, and lymphoid cells, indicating that IL-1 $\beta$ expression in neutrophils and eosinophils is a common signature of pediatric granulocytes in the setting of inflammation.

Altered maturation and increased activation of neutrophils in KD and MIS-C. To understand the phenotypic and functional chang- 
Table 1. Comparison of demographic and clinical characteristics, treatment, and outcome among disease cohorts

\begin{tabular}{|c|c|c|c|c|c|c|c|}
\hline \multirow[t]{2}{*}{ Characteristic } & KD & MIS-C & $\mathrm{FC}$ & \multirow[t]{2}{*}{$P^{A}$} & \multirow[t]{2}{*}{$P^{B}$} & \multirow[t]{2}{*}{$P^{C}$} & \multirow[t]{2}{*}{$P^{D}$} \\
\hline & $n=11$ & $n=15$ & $n=24$ & & & & \\
\hline Male, $n(\%)$ & $6(54.5)$ & $10(66.7)$ & $14(58.3)$ & 0.802 & & & \\
\hline Female, $n(\%)$ & $5(45.5)$ & $5(33.3)$ & $10(41.7)$ & & & & \\
\hline Illness day, median (IQR) & $6.0(3.0-7.0)$ & $5.0(3.0-5.0)$ & $5.0(2.0-5.0)$ & 0.073 & 0.183 & 0.064 & 0.918 \\
\hline Elllness day at subacute visit, median (IQR) & $22.0(19.0-26.0)$ & $25.0(19.0-27.0)$ & - & 0.434 & & & \\
\hline \multicolumn{8}{|l|}{ Ethnicity, $n$ (\%) } \\
\hline Black & $0(0)$ & $1(6.7)$ & $1(4.2)$ & 0.692 & & & \\
\hline White & $5(45.5)$ & $1(6.7)$ & $8(33.3)$ & 0.068 & & & \\
\hline Hispanic & $4(36.4)$ & $10(66.7)$ & $12(50.0)$ & 0.300 & & & \\
\hline More than one race & $1(9.1)$ & $3(20.0)$ & $2(8.3)$ & 0.521 & & & \\
\hline \multicolumn{8}{|l|}{ Laboratory data, median (IQR) } \\
\hline WBC $\left(10^{3} / \mu \mathrm{L}\right)$ & $14.1(10.3-16.9)$ & $10.9(5.1-14.3)$ & $9.8(5.6-15.9)$ & 0.172 & 0.178 & 0.246 & 0.922 \\
\hline Platelets $\left(10^{3} / \mu \mathrm{L}\right)$ & $269.0(215.0-434.0)$ & $153.0(108.0-236.0)$ & $239.0(181.3-362.8)$ & 0.006 & 0.008 & 0.561 & 0.028 \\
\hline $\operatorname{ESR}(\mathrm{mm} / \mathrm{h})$ & $40.0(29.0-40.0)$ & $48.0(27.0-81.0)$ & $18.5(10.3-53.0)$ & 0.095 & 0.626 & 0.586 & 0.079 \\
\hline $\operatorname{CRP}(\mathrm{mg} / \mathrm{dL})$ & $6.1(4.2-8.5)$ & $20.7(15.8-36.3)$ & $2.2(0.6-6.3)$ & 0.0001 & 0.004 & 0.968 & 0.0001 \\
\hline $\operatorname{ALT}(\mathrm{U} / \mathrm{L})$ & $30.0(18.0-98.0)$ & $40.0(21.0-65.0)$ & $16.5(13.3-22.8)$ & 0.010 & 0.916 & 0.024 & 0.039 \\
\hline GGT (U/L) & $22.0(13.0-80.0)$ & $33.0(25.8-90.3)$ & $14.0(13.0-18.5)$ & 0.003 & 0.531 & 0.067 & 0.004 \\
\hline Sodium (mmol/L) & $134.0(131.0-137.0)$ & $132.0(128.0-133.0)$ & $133.5(132.0-136.0)$ & 0.022 & 0.035 & 0.823 & 0.048 \\
\hline Albumin (g/dL) & $3.4(3.1-3.8)$ & $3.5(3.3-4.1)$ & $4.2(3.9-4.4)$ & $<0.0001$ & 0.965 & 0.0006 & 0.0004 \\
\hline $\mathrm{BNP}(\mathrm{pg} / \mathrm{mL})^{\mathrm{H}}$ & - & $661.0(30.0-2081.0)$ & - & - & & & \\
\hline Ferritin $(\mathrm{ng} / \mathrm{mL})^{\mathrm{H}}$ & - & 707.0 (319.0-1179.0) & - & - & & & \\
\hline Troponin $(\mathrm{ng} / \mathrm{mL})^{\mathrm{H}}$ & - & $0.04(0.01-0.43)$ & - & - & & & \\
\hline \multicolumn{8}{|l|}{ Treatment, $n(\%)$} \\
\hline \multicolumn{8}{|c|}{ 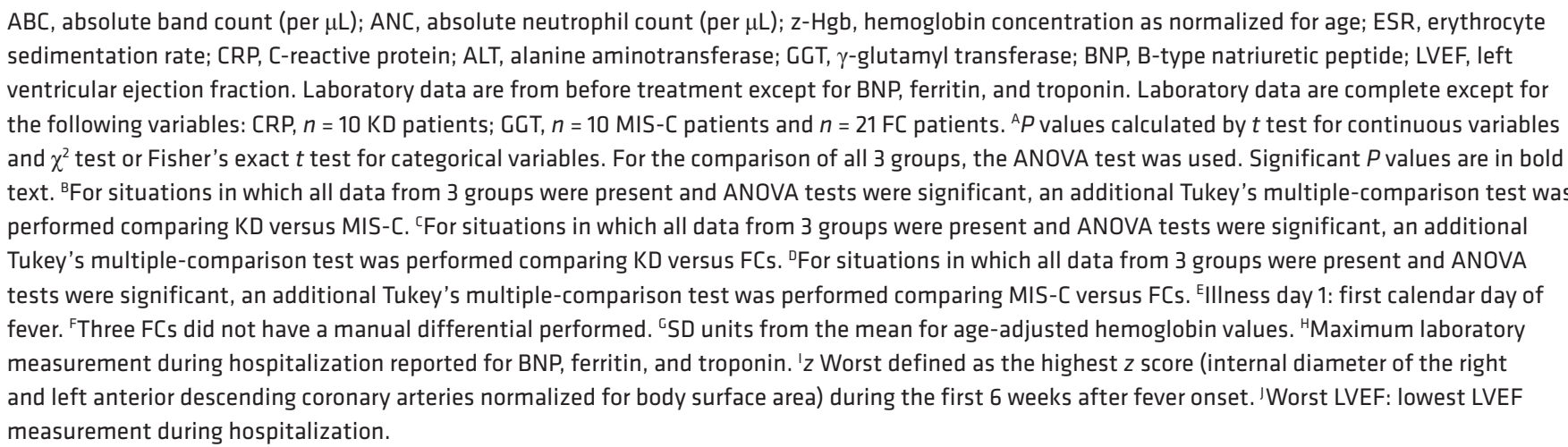 } \\
\hline
\end{tabular}

es occurring in neutrophils from patients with KD and MIS-C, we designed a CyTOF panel that discriminated major myeloid and lymphoid lineages and interrogated markers indicative of granulocyte development, migration, adhesion, activation, and inflammation (Table 2 and Supplemental Figure 2A). Dimensional reduction and automated clustering analysis of the peripheral blood CyTOF data from 9 MIS-C patients, $2 \mathrm{KD}$ patients, and 11 FC patients demonstrated that leukocyte lineages were clearly discriminated with this approach while also providing enhanced resolution of neutrophil, monocyte, eosinophil, and natural killer cell heterogeneity in the 3 cohorts (Figure 3A and Supplemental Figure 2, B-D). Consistent with the flow cytometry results, we observed 

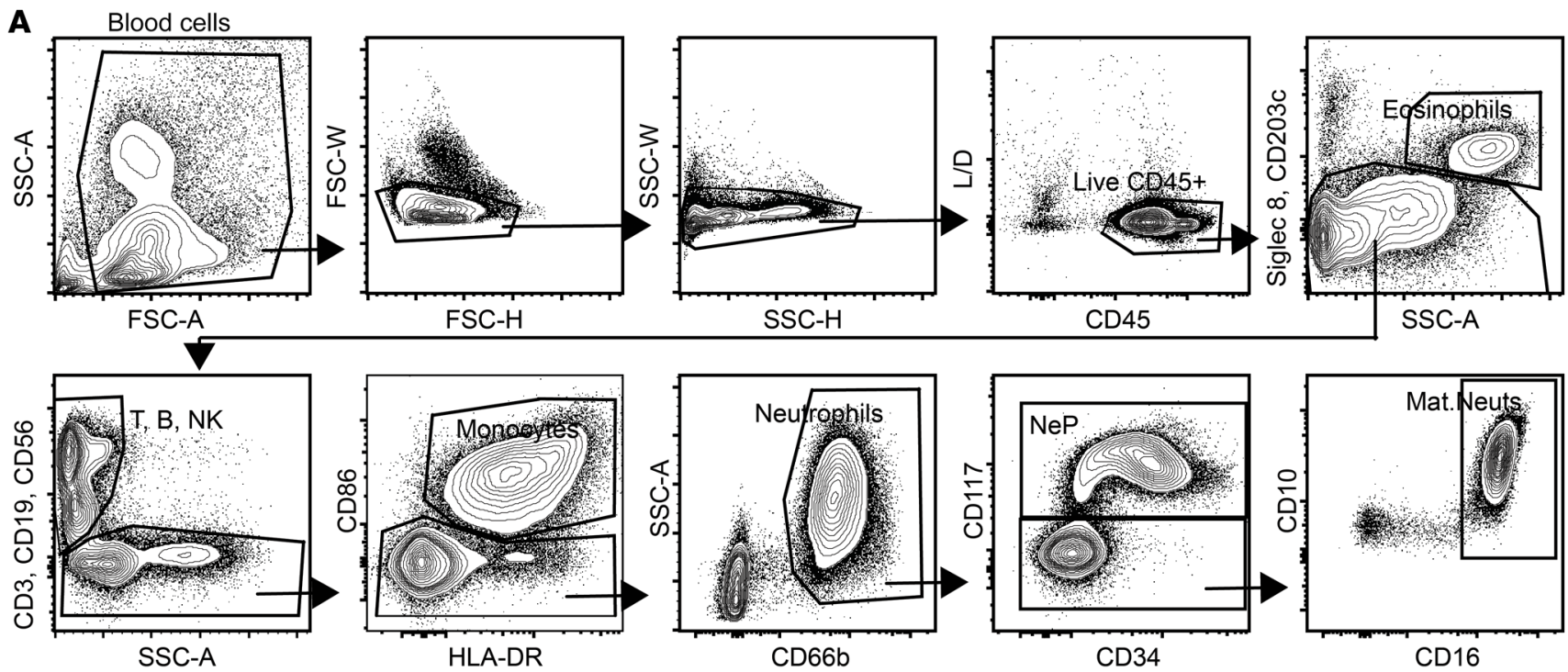

B

T, B, NK

HLA-DR

CD66b

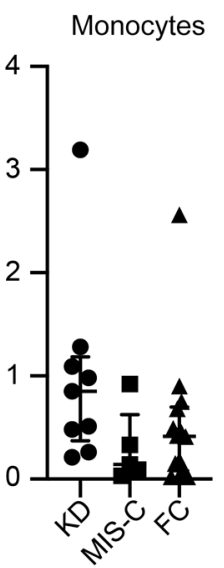

Eosinophils

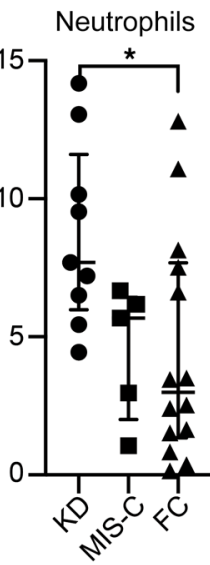

C

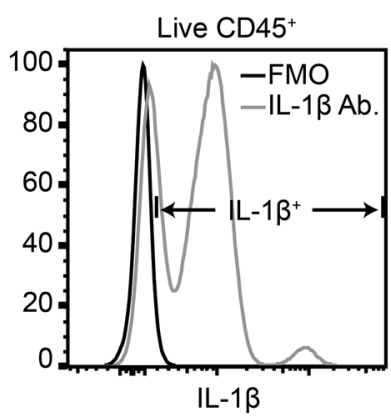

D IL-1 $\beta^{+}$cells

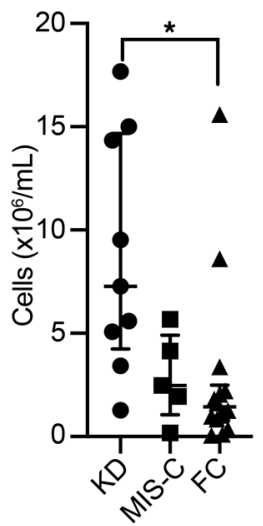

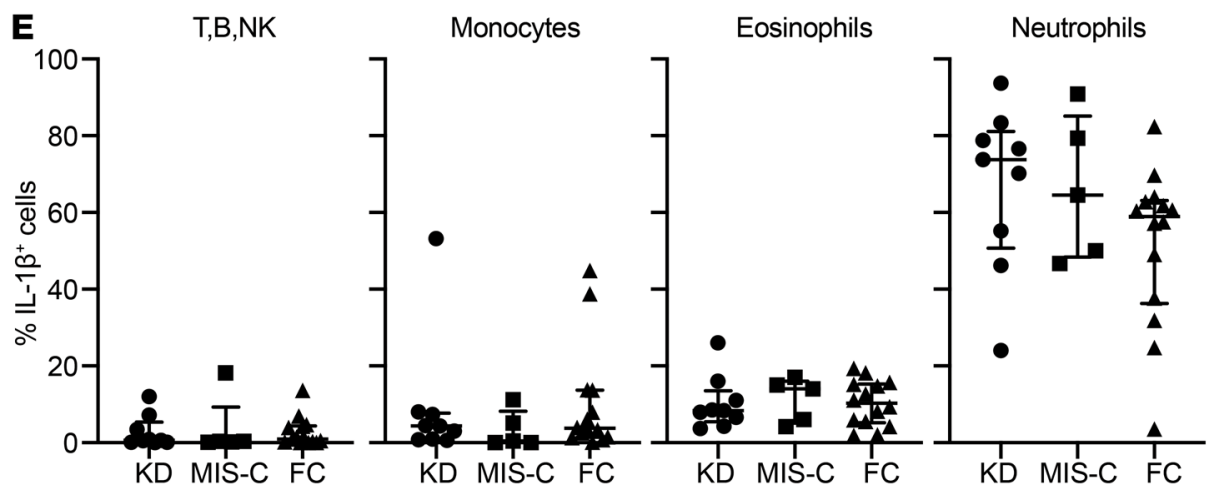

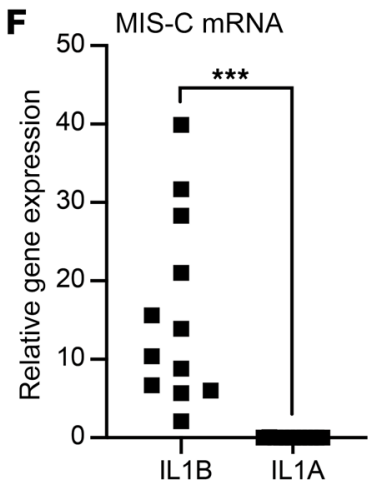

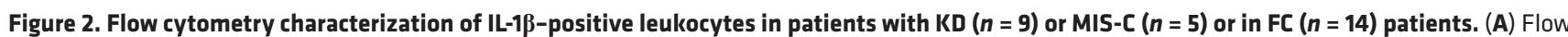
cytometry gating strategy used to distinguish myeloid and lymphoid cells. (B) Dot plots show the total cell numbers of each cell population per mL of whole blood from all patients prior to treatment. (C) Flow cytometry gating strategy for this study demonstrating IL-1 $\beta^{+}$expression in each population. Fluorescence minus one (FMO) controls were used for each sample to assist gating. (D) Flow cytometry evaluation of absolute cell numbers of IL-1 $\beta^{+}$ leukocytes per $\mathrm{mL}$ of whole blood from all patients prior to treatment. (E) Scatter dot plots show each IL-1 $\beta^{+}$cell population as a percentage of all IL-1 $\beta^{+}$ leukocytes from all patients prior to treatment. (F) qPCR mRNA expression of indicated genes in neutrophils isolated from 12 MIS-C patients prior to treatment. $P$ values were determined by 1-way ANOVA and Tukey's multiple-comparison tests with a single pooled variance. Horizontal lines on dot plots indicate median IQRs. Mat.Neuts, mature neutrophils. ${ }^{*} P<0.05,{ }^{* * *} P<0.0005$. 
A
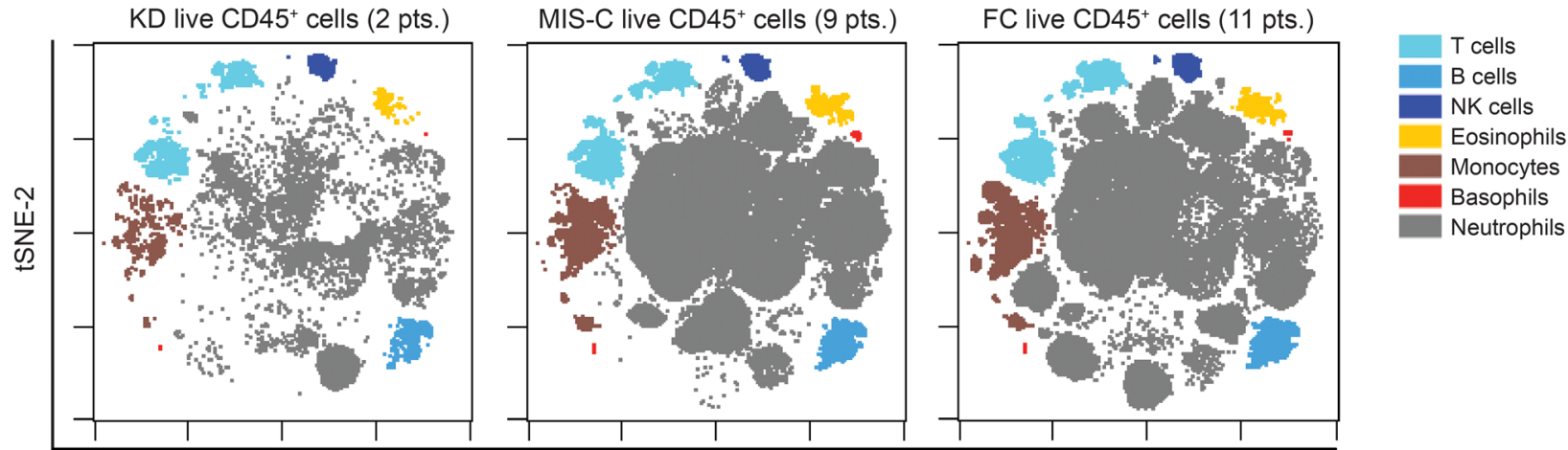

tSNE-1

B

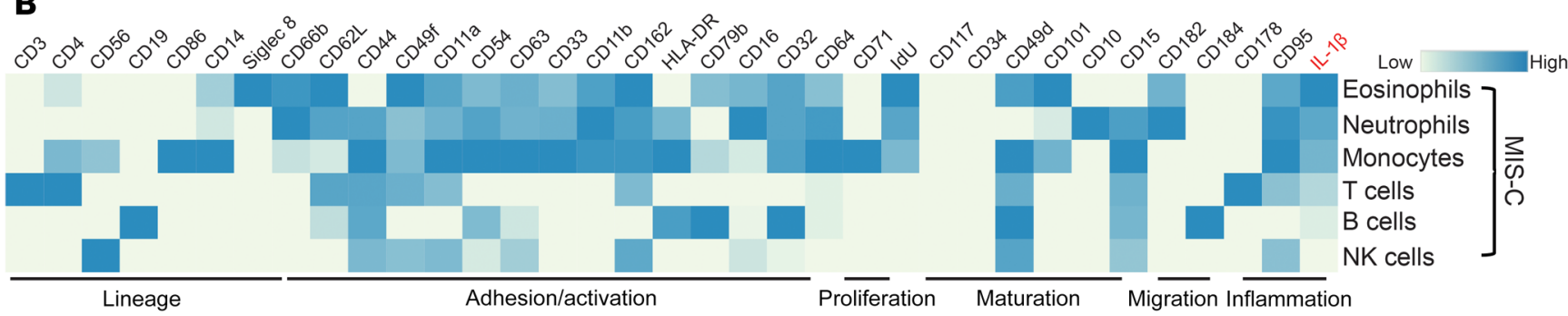

\section{C}
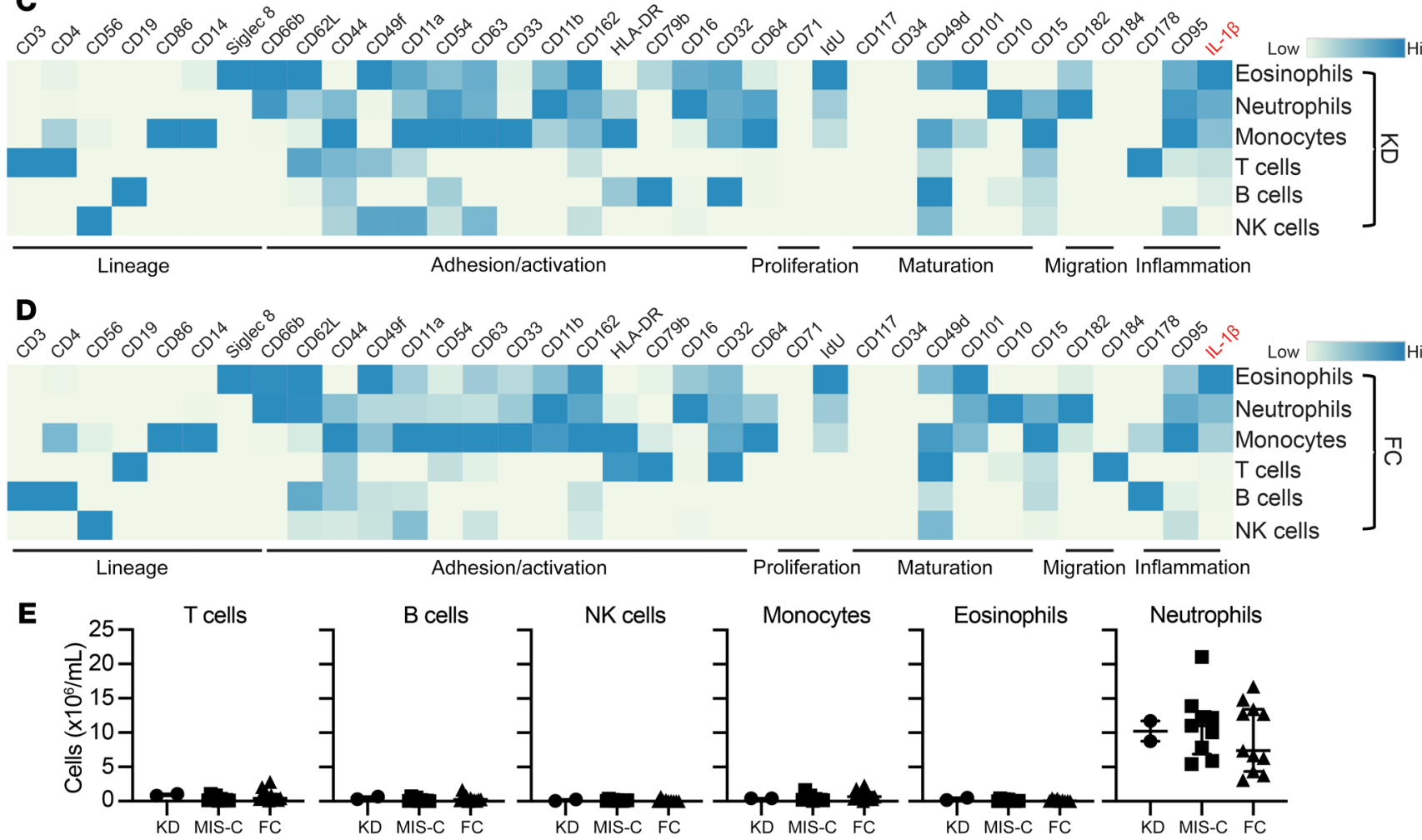

Figure 3. CyTOF shows neutrophils are highly activated in patients with KD or MIS-C and associated with high IL-1 1 expression. (A) viSNE map shows FLOWSOM automated clustering results of live $\mathrm{CD}^{4} 5^{+}$cells as leukocyte lineage populations from all patients prior to treatment. (B) Heatmap shows the median metal intensity of each marker in different leukocyte populations from patients with MIS-C (B, $n=9), K D(C, n=2)$, and FCs (D, $n=11)$ obtained before treatment. (E) Total cell numbers of leukocyte populations prior to treatment from patients with KD $(n=2)$, MIS-C ( $n=9)$, or from FCs $(n=11)$. Values determined from CyTOF data. $P$ values were determined by 1-way ANOVA and Tukey's multiple-comparison tests with a single pooled variance. Lines on scatter dot plots indicate median with IQR. tSNE, $t$ distributed stochastic neighbor embedding; viSNE, visualization of $t$ distributed stochastic neighbor embedding. 
IL-1 $\beta$ expression in eosinophils, neutrophils, and monocytes in all 3 cohorts (Figure 3, B-D). Neutrophils from MIS-C patients expressed the highest levels of activation markers compared with KD and FC groups. No significant differences were evident in lymphoid and myeloid counts among MIS-C, KD, and FCs (Figure 3E and Supplemental Figure 2E); however, neutrophilia was evident in all cohorts, suggesting that neutrophils are a substantial source of IL-1 $\beta$ in these patients.

We then focused on the specific phenotypes of neutrophils in the 3 patient cohorts. CyTOF showed distinct marker-expression profiles of neutrophil lineage cells (automated Siglec-8-negative CD66b-enriched clusters) in each cohort (Figure 4A). The neutrophil lineage from MIS-C patients was characterized by increased markers of adhesion and activation, including CD62L, CD44, CD54, CD63, and HLA-DR when compared with FCs (Figure 4A). In contrast with those from FCs, neutrophil lineage cells from KD patients exhibited elevated CD44, CD11a, CD54, CD63, HLA-DR, and CD64, reduced CD66b, and activation marker profiles similar to those of MIS-C cells, including CD54 and CD63. Maturation markers, including CD49d, CD101, and CD10, were more abundant in FCs than in KD and MIS-C neutrophil lineage cells. The IL-8 receptor $\beta$ chain (CD182) was most abundant on neutrophil lineage cells from FC patients and least abundant on MIS-C cells. The Fas death receptor (CD95) was abundantly expressed on cells from KD and MIS-C patients. Differential expression of IL-1 $\beta$ was also evident in neutrophil lineage cells between the cohorts, with highest expression detected in KD patients. These results suggest that neutrophils from KD and MIS-C are immature and highly activated.

To further study the major effects of IVIG on blood leukocytes, particularly neutrophil lineage cells, we used the flow cytometry gating strategy shown in Figure 2A to examine the peripheral blood of patients with MIS-C and KD prior to IVIG administration and 2 to 6 weeks afterwards. Results demonstrated that in all leukocyte populations, IVIG treatment was associated with a significant reduction in neutrophil counts in KD samples and a more than $50 \%$ reduction in neutrophil lineage cells in $\mathrm{KD}$ and MIS-C samples (Figure 4B). This reduction in neutrophil numbers was accompanied by an increased lymphocyte proportion in both cohorts (Figure 4B). In contrast, there was no significant change in the absolute numbers of monocytes. Eosinophil numbers were not significantly affected by IVIG treatment. IVIG administration was common to all patients in this study; however, effects of adjunctive infliximab and/or corticosteroids cannot be discounted. Of the patients studied 2 to 6 weeks after IVIG treatment, 7 of $8 \mathrm{KD}$ patients and 4 of 9 MIS-C patients received only IVIG. The treatment regimens of the remaining patients are indicated (Figure 4B). The reduction in neutrophil numbers in KD patients 2 to 6 weeks after IVIG-only treatment was consistent with data from an independent KD cohort that demonstrated a sharp decline in neutrophil numbers 24 hours after IVIG treatment (Figure 4C). These data support a direct role for IVIG on neutrophils that was most pronounced in KD patients, although effects on other immune cell types may also contribute to disease resolution.

$I L-1 \beta^{+}$neutrophils are greatly reduced after IVIG treatment. Next, we examined intracellular IL-1 $\beta$ expression in patients with KD and MIS-C in the subacute phase (2 to 6 weeks after IVIG treatment). IL-1 $\beta$-expressing leukocytes were reduced in number in the sub- acute phase in KD cohorts (Figure 4D and Supplemental Figure $3)$. Given that most IL-1 $\beta$-expressing leukocytes were neutrophils (Figure 2E), we investigated the IL-1 $\beta$ expression in neutrophils from these cohorts. Results showed that IVIG treatment was associated with reduced IL- $1 \beta$ production in neutrophils in both cohorts (Figure 4E) and was associated with a more than $90 \%$ reduction in IL-1 $\beta$-expressing neutrophils in the circulation (Figure $4 \mathrm{~F}$ ). This effect was specific to neutrophils, as we did not observe changes in all other IL-1 $\beta^{+}$leukocyte populations (Figure $4 \mathrm{~F}$ ). The disappearance of IL-1 $\beta$-expressing neutrophils was observed in all $8 \mathrm{KD}$ patients who received IVIG alone and also in the 4 MIS-C patients who received only IVIG and no other adjunctive therapy.

We then focused on neutrophil lineage subpopulations to further understand the changes in IL-1 $\beta$-expressing cell numbers following IVIG treatment. Recent studies have demonstrated heterogeneity in blood neutrophils, with different developmental stages present in the setting of acute inflammation. Neutrophil progenitors (NePs), which correspond to the promyelocyte stage of differentiation, can be distinguished by coexpression of the stem cell markers CD117 and CD34 as well as the neutrophil marker CD66b $(29,30)$. We observed a significant reduction of mature neutrophils in both KD and MIS-C patients and reduced NeP counts in KD patients following IVIG treatment (Figure 5, A and B, and Supplemental Figure 4, A and B). The majority of the mature neutrophils were IL- $1 \beta^{+}$, and IVIG treatment was associated with a reduction of granulocyte colony-stimulating factor (G-CSF) levels and IL- $1 \beta^{+}$mature neutrophils in patients with $\mathrm{KD}$ (Figure 5, C and D). We also observed variable numbers of IL-1 $\beta$-expressing NePs in both KD and MIS-C patients, but this population was not significantly altered following IVIG treatment (Figure 5E). No changes were detected in total numbers of IL-1 $\beta$-negative mature neutrophils and NePs following IVIG treatment. Thus, IVIG appears to target specifically mature IL- $1 \beta^{+}$neutrophils.

To explore the acute changes elicited by IVIG in the neutrophil lineage cells from MIS-C patients up to 30 hours after treatment in the absence of adjunctive immunotherapy, we analyzed the phenotypic profile of neutrophil lineage cells from CyTOF data. IL-1 $\beta$ expression was reduced following IVIG treatment, consistent with flow cytometry data (Figure 5F). Activation and adhesion markers, including CD62L, CD11a, CD54, CD63, CD33, CD11b, HLA-DR, CD32, and CD64, were also reduced following IVIG treatment. Increases in CD49f, CD162, CD79b, and CD16 were evident following IVIG. Changes in maturation status associated with IVIG treatment included an increase in CD49d and CD10 and a decrease in CD101 and CD15 (Figure 5F). The changes in CD10 and CD16 detected by CyTOF are consistent with the changes in cell-surface expression detected by flow cytometry (Figure 5A). Thus, IVIG has pleiotropic effects on both neutrophil maturation and cell-surface marker expression.

To identify specific neutrophil subsets targeted by IVIG treatment, we further investigated the heterogeneity of neutrophil lineage cells in CyTOF data sets by performing a secondary automated clustering technique based on Siglec-8-negative CD66b-enriched neutrophil lineage clusters. The results revealed at least 5 populations, including 3 mature neutrophil subsets and $2 \mathrm{NeP}$ subsets, that have been previously described $(30,31)$. These subsets displayed differential expression of IL-1 $\beta$ levels (Figure $5 G$ ). Higher IL-1 $\beta$ 
A

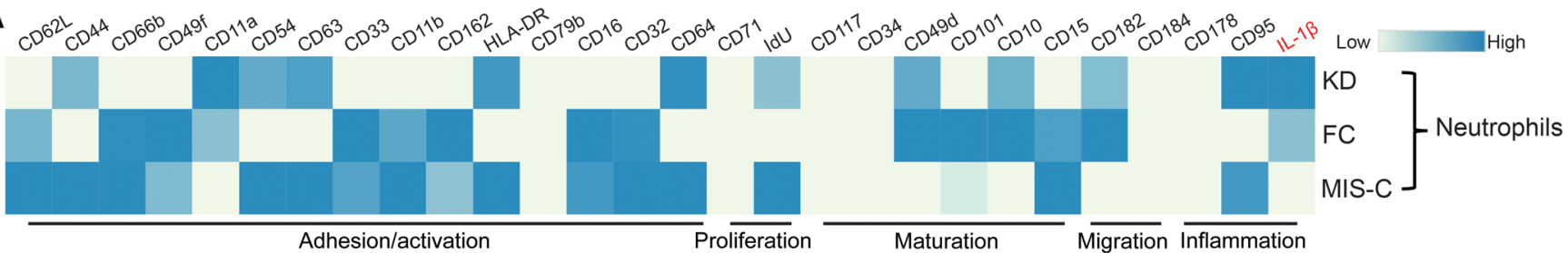

B

T,B,NK

Monocytes

Eosinophils

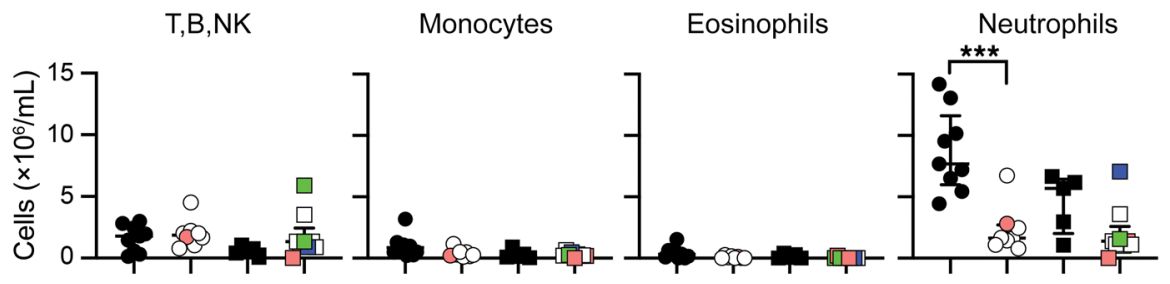

C

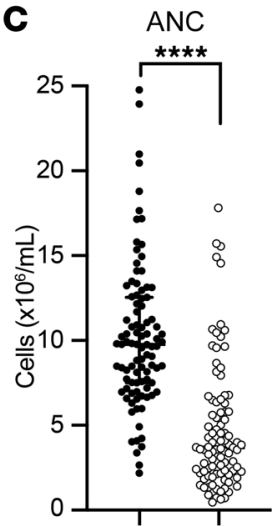

D

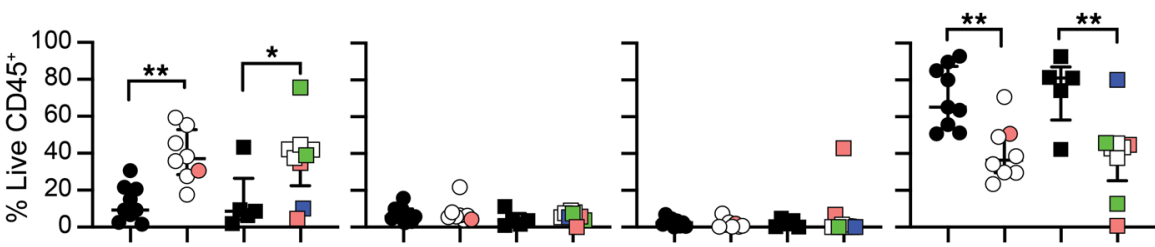

MIS-C neutrophils

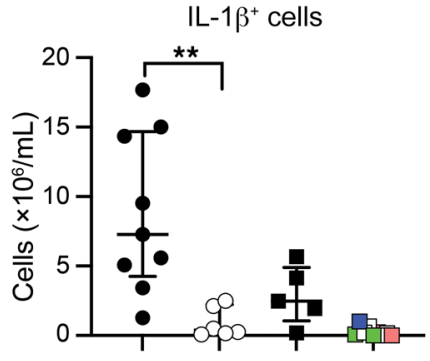

$\mathbf{F}$

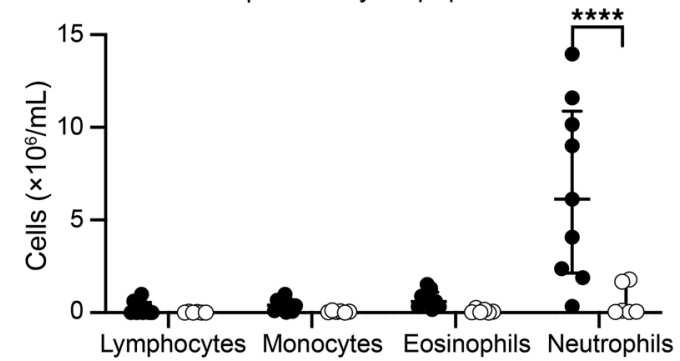

KD neutrophils

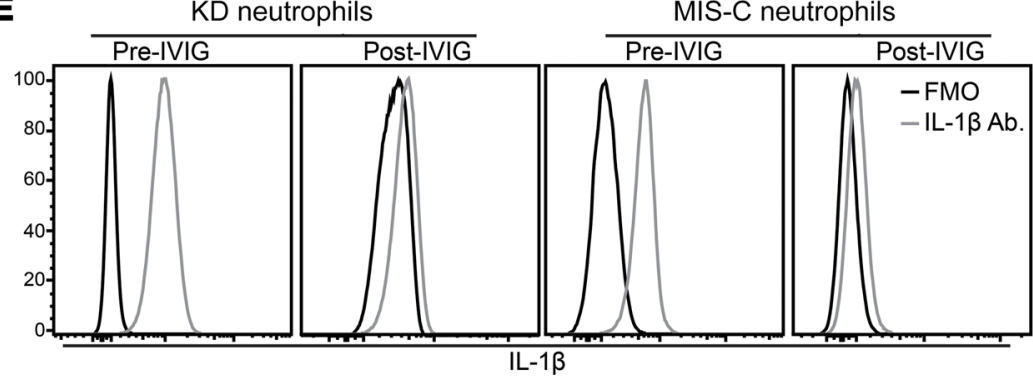

MIS-C IL-1 $\beta^{+}$leukocytes populations

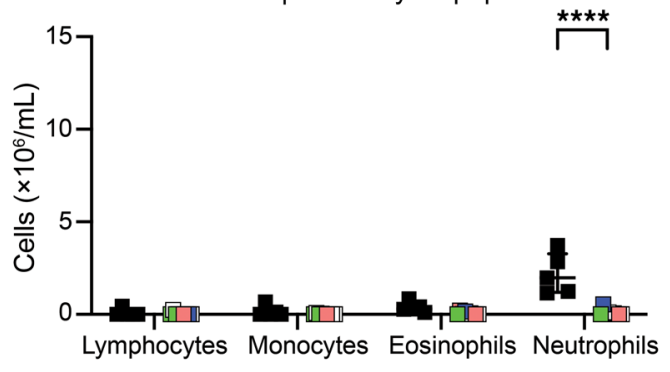

- KD, pre-IVG

- MIS-C, pre-IVG

KD, post-IVG (IVIG alone; IVIG + Infliximab;IVIG + Infliximab + steroids; IVIG + steroids)

$\square$ MIS-C, post-IVG (IVIG alone; IVIG + Infliximab;IVIG + Infliximab + steroids;IVIG + steroids)

Figure 4. IVIC treatment in patients with KD or MIS-C is associated with a decrease in IL-1ß-producing neutrophils and an increase in lymphocytes. (A) Heatmap shows the median metal intensity of each marker in neutrophils from all patients before treatment. (B) Total cell number (top panels) and proportion (bottom panels) of leukocyte populations from patients with KD $(n=9)$ or MIS-C $(n=5)$ prior to IVIG treatment and 2 to 6 weeks after treatment (KD, $n=8$; MIS-C, $n=9$ ). Values determined from flow cytometry data. (C) Absolute neutrophil count (ANC) from KD patients before IVIC and 24 hours after IVIC treatment from an independent cohort $(n=95)$. (D) Total numbers of IL-1 $\beta^{+}$leukocytes from KD patients prior to treatment $(n=9)$ and 2 to 6 weeks after IVIC $(n=8)$. (E) IL-1 $\beta$ expression in neutrophils from a patient with KD and a patient with MIS-C prior to treatment and 2 to 6 weeks after IVIG. Representative data shown. Fluorescence minus one of each sample was used as a staining control. (F) Total numbers of IL-1 $1 \beta^{+}$lymphocytes, monocytes, eosinophils, and neutrophils in the peripheral blood of patients with KD (left panel) or MIS-C (right panel) determined by flow cytometry before treatment (KD, $n=9$; MIS-C, $n=5$ ) and 2 to 6 weeks after IVIC treatment (KD, $n=8$; MIS-C, $n=9$ ). $P$ values were determined by unpaired $t$ tests based on IVIG-alone samples. Lines on dot plots indicate median with IQR. ${ }^{*} P<0.05 ;{ }^{* *} P<0.005 ;{ }^{* *} P<0.0005 ;{ }^{* * *} P<0.0001$. 

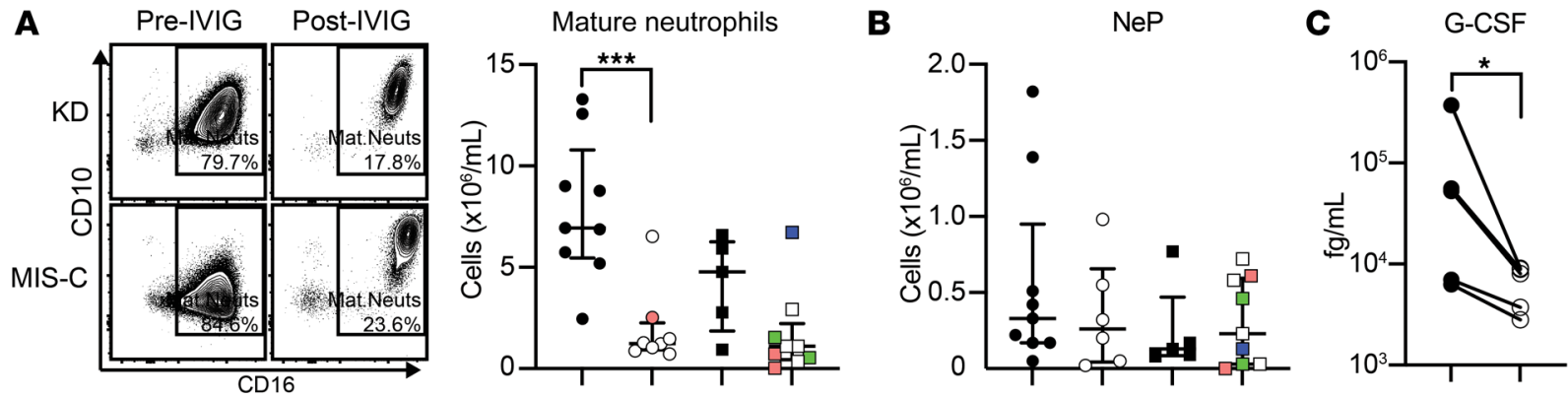

D $\quad \mathrm{L}-1 \beta^{+}$mature $\quad \mathrm{IL}-1 \beta^{-}$mature

E

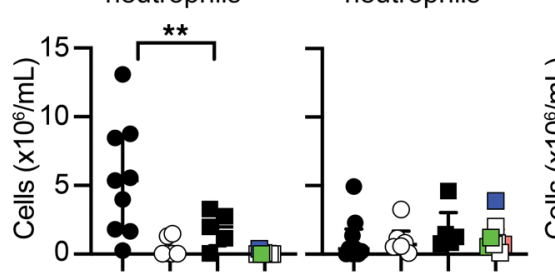

IL-1 $\beta^{+} \mathrm{NeP}$

IL- $1 \beta^{-} \mathrm{NeP}$

- KD, pre-IVIG
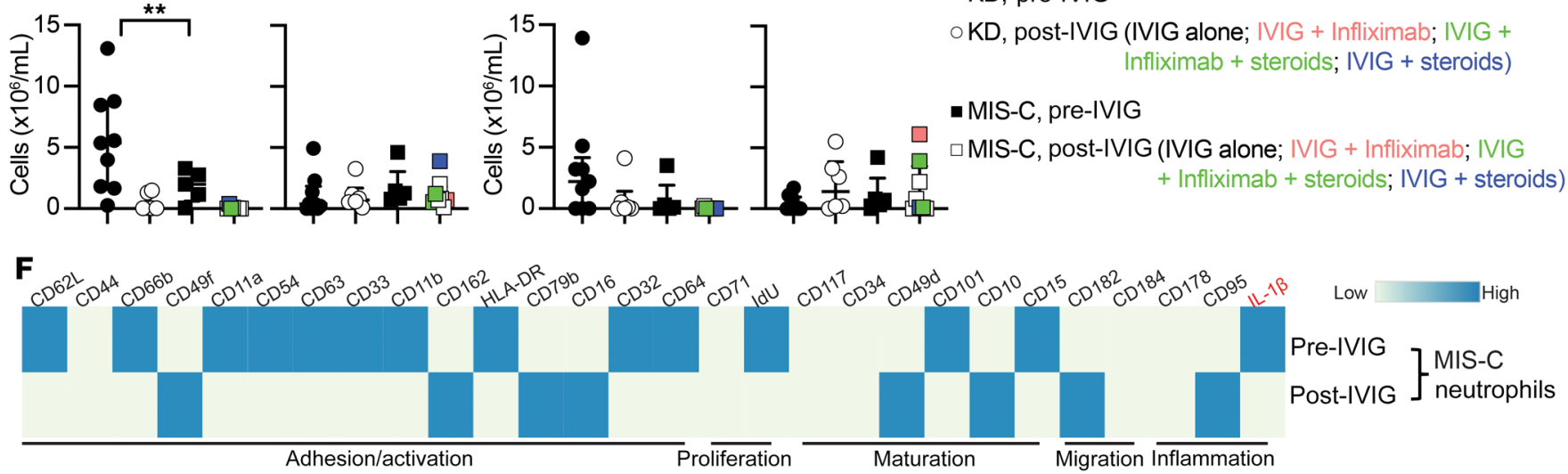

G
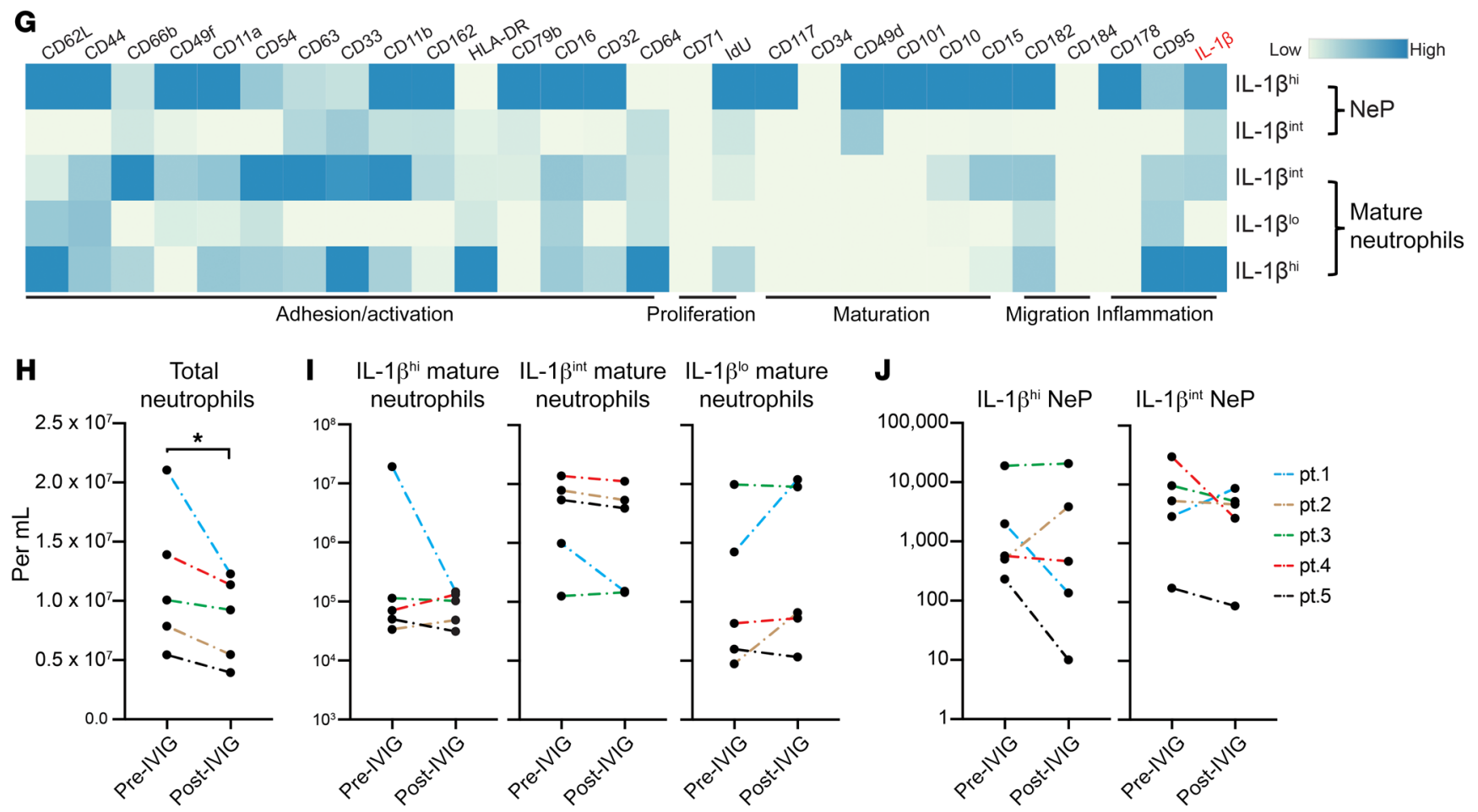

I IL-1 $\beta^{\text {hi }}$ mature

IL-1 $\beta^{\text {int }}$ mature IL-1 $\beta^{\text {lo }}$ mature
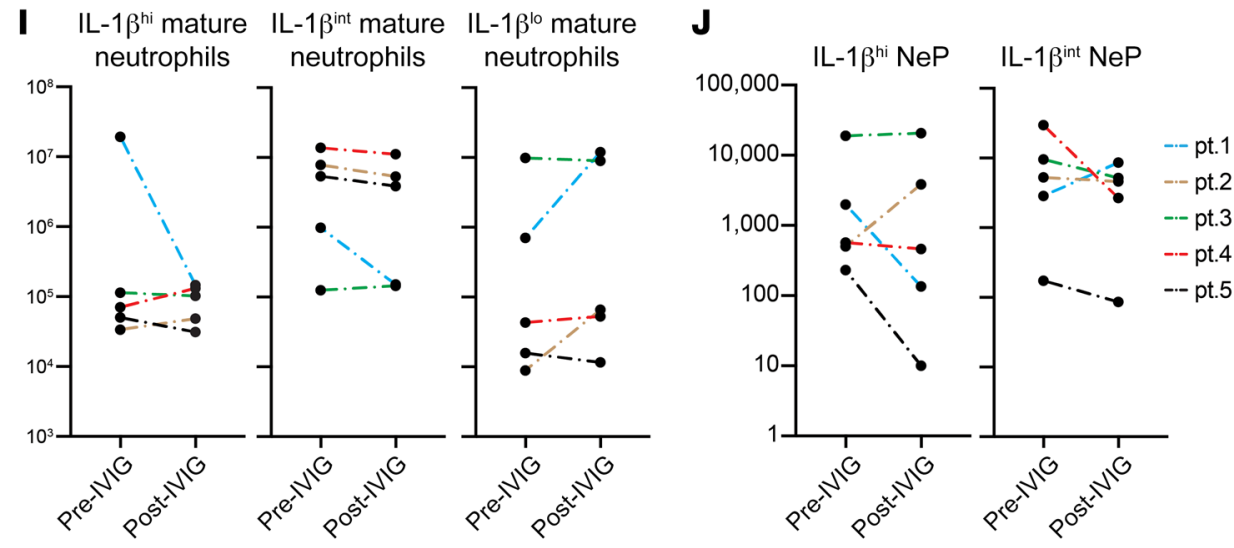
Figure 5. IVIC treatment in patients with KD or MIS-C is associated with

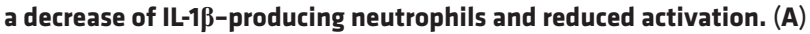
Left panel: flow cytometry gating strategy demonstrating reduced mature neutrophils after IVIC treatment in a representative KD patient (top) and a representative MIS-C patient (bottom). The percentages of neutrophils in live $\mathrm{CD} 45^{+}$cells are indicated on the contour plots. Right panel: absolute cell numbers of mature neutrophils per $\mathrm{mL}$ of whole blood. (B) Flow cytometry evaluation of NePs per $\mathrm{mL}$ of whole blood. (C) Serum G-CSF in patients with KD before treatment $(n=5)$ and 2 to 6 weeks after IVIC treatment $(n=5)$. (D) Flow cytometry evaluation of total numbers of IL-1 $\beta^{+}$ mature neutrophils (left panel) and IL-1 $\beta^{-}$mature neutrophils (right panel) per $\mathrm{mL}$ of whole blood. (E) Flow cytometry evaluation of total numbers of IL-1 $1 \beta^{+} \mathrm{NePs}$ (left panel) and IL-1 $1 \beta^{-} \mathrm{NePs}$ per $\mathrm{mL}$ of whole blood (right panel). (F) Heatmap shows the median metal intensity of each marker in neutrophils from 5 MIS-C patients before treatment and 1 day after IVIG treatment evaluated by CyTOF. (G) Heatmap showing FLOWSOM automated clustering of neutrophil subsets from 5 MIS-C patients before treatment and 1 day after IVIG treatment. (H-J) CyTOF evaluation of total cell numbers of neutrophils (H), mature neutrophil subsets (I), and NeP subsets (J) per $\mathrm{mL}$ of whole blood. Samples were analyzed from 5 patients with MIS-C prior to treatment and 1 day after IVIG treatment. Differences between before and after IVIC treatment were determined via unpaired $t$ tests (2-tailed) based on IVIG-alone samples (A, B, D, and E) or by ratio paired $t$ tests (C, $\mathbf{H}, \mathbf{I}$, and $\mathbf{~})$. Lines on dot plots indicate median with IQR. ${ }^{*} P<0.05 ;{ }^{* *} P<0.005 ;{ }^{* *} P<0.0005$

expression levels were associated with elevated activation status in neutrophil subsets. The total numbers of cells in the neutrophil lineage were reduced in all 5 MIS-C patients after receiving IVIG treatment (Figure $5 \mathrm{H}$ ). Within the total population of cells in the neutrophil lineage, the activated, IL-1 $\beta$-intermediate subset was reduced compared with the less activated, IL-1 $\beta$-low subset and NeP subsets (Figure 5, I and J, and Supplemental Figure 4C). These high-dimensional CyTOF analyses demonstrate rapid changes in leukocyte populations induced by IVIG treatment and a complexity to neutrophil maturation in these disease contexts that may reflect neutrophil subset specialization in function and activation.

IVIG induces neutrophil cell death independently of apoptosis. Neutrophil death has been described in different animal model and clinical settings as occurring through apoptotic and nonapoptotic pathways. Previous neutrophil studies with IVIG have attributed the loss of cell viability to an apoptotic mechanism (32-35). To characterize the biochemical pathway through which IVIG was killing neutrophils in MIS-C and KD patients, we used inhibitors of apoptosis and caspase-1-dependent pyroptosis (QVD-OPh), necroptosis (Nec-1s), ferroptosis (liproxstatin-1 and ferrostatin-1), and NETosis (BBCl-Amidine, GSK199, GSK484) as well as inhibitors of PI3K (PIK-75 and GDC-0941), NADPH oxidase (DPI), and mitochondrial reactive oxygen species (MitoTEMPO). In experiments with neutrophils harvested from acute, pretreatment MIS-C and KD patients and exposed ex vivo to IVIG, we observed a loss of cell viability characterized by staining with annexin $\mathrm{V}$ (AnnV) and propidium iodide (PI) (Figure 6A). To investigate the biochemical pathways involved in IVIG signaling in neutrophils, we used live-cell imaging to examine the morphological and kinetic changes in neutrophils induced by IVIG and inhibitors described above. To enable accurate segmentation of objects in live-cell imaging, we incorporated a deep learning module called StarDist into custom-scripted code to allow trainable neural net- works to analyze images instead of traditional watershed segmentation (36). This approach allowed changes in cell populations to be classified according to the cell death markers AnnV, which binds externalized phosphatidylserine of dying cells, and PI, which binds DNA of cells with compromised plasma and nuclear membranes. IVIG-triggered cell death was blocked by the PI3K inhibitors PIK-75 and GDC-0941 and also by the NADPH oxidase inhibitor DPI (Figure 6B). Cell death was not inhibited by the pancaspase inhibitor Q-VD-OPh, suggesting that IVIG was not triggering cell death via apoptosis signaling or via caspase-1-dependent pyroptosis (Figure 6B). IVIG was not engaging cell death pathways via RIPK1, ferroptosis, NETosis, or mitochondrial reactive oxygen species (Supplemental Figure 5A). Q-VD-OPh was able to impair cell death induced by Fas ligand (FasL) (Supplemental Figure 5B). FasL was used as a control to induce apoptosis and resulted in an equivalent reduction in viability compared with IVIG (Figure 6B). These findings were consistent for neutrophils isolated from acute KD and MIS-C patients, suggesting a similar mechanism of action of IVIG in both diseases that occurs via a pathway independent of apoptosis, pyroptosis, necroptosis, and ferroptosis.

Live-cell imaging confirms neutrophil cell death by a nonapoptotic pathway. Using custom-scripted code to identify objects in livecell imaging data coupled to a data visualization tool called DeathTrackR, we examined cell death signatures from neutrophils treated with IVIG or FasL (Figure 6C). DeathTrackR is a user-directed visualization tool, built on the R Shiny package, that allows import of csv files generated from automated analysis of live-cell imaging data sets to visualize cell death transition states during the cell death process. Neutrophils treated with IVIG transformed rapidly from viable CellTracker Green $(\mathrm{CTG})^{+} \mathrm{AnnV}^{-} \mathrm{PI}^{-}$cells to dead $\mathrm{CTG}^{+} \mathrm{AnnV}^{+} \mathrm{PI}^{+}$cells (Figure $6 \mathrm{C}$ ). In contrast, neutrophils treated with FasL generated an apoptotic $\mathrm{CTG}^{+} \mathrm{AnnV}^{+} \mathrm{PI}^{-}$state prior to transition to a dead $\mathrm{CTG}^{+} \mathrm{AnnV}^{+} \mathrm{PI}^{+}$state (Figure $6 \mathrm{C}$ ). When FasL was added to IVIG, the pathway activated by IVIG was dominant to the effects of the Fas-dependent apoptotic-signaling cascade (Figure 6C). Purified Fc did not replicate the actions of IVIG in human neutrophils (Figure 6C), consistent with previous reports (37). This confirmed that $\mathrm{F}\left(\mathrm{ab}^{\prime}\right)_{2}$ was responsible for triggering neutrophil death. By analyzing the size of individual cells in these live-cell imaging data sets, it was evident that IVIG was also triggering an increase in cell size as well as causing a loss of viability (Figure 6D). These data are consistent with time-lapse images of human neutrophils treated with IVIG, FcFasL, or PBS (Supplemental Videos 1, 2, and 3, respectively). These live-cell data sets support a mode of cell death distinct from apoptosis.

Dimension reduction of live-cell imaging to compare IVIG and FasL responses. To simultaneously analyze morphological and fluorescence features from these time-lapse files, we performed a dimension reduction analysis using the nonlinear dimension reduction algorithm UMAP (38). These analyses generate 2D representations of individual cellular relationships according to cell size, cell aspect ratio, and fluorescence intensity of CTG, AnnV, and PI (Figure 7, A and B). Neutrophils treated with IVIG clustered in distinct locations compared with cells treated with the apoptotic stimulus FasL (Figure 7, A and B) despite similar kinetics of cell death (Figure 6C). These differences between IVIG and FasL were apparent when examining both morphological and fluorescence 
A
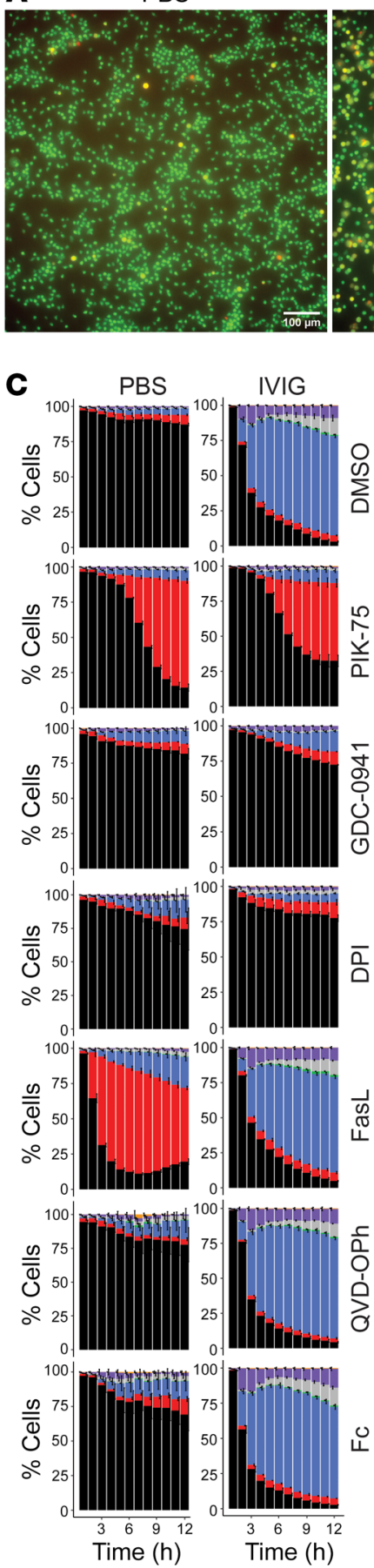

$1 \%$ IVIG

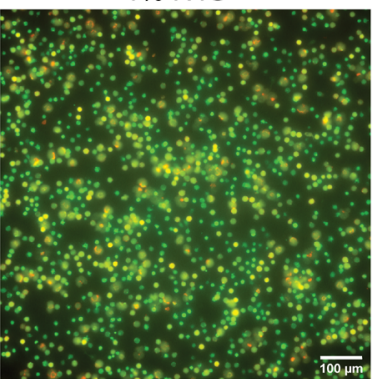

CTTG-AnnV-PI+

$\mathrm{CTG}^{+} \mathrm{AnnV}^{-\mathrm{PI}} \mathrm{I}^{+}$

CTG-AnnV+PI-

CTG-AnnV+PI+

CTG $^{+} \mathrm{AnnV}^{+} \mathrm{Pl} \mathrm{I}^{+}$

$\mathrm{CTG}^{+} \mathrm{AnnV}^{+} \mathrm{PI}$

$\mathrm{CTG}^{+} \mathrm{AnnV}^{-\mathrm{P}} \mathrm{I}^{-}$
B

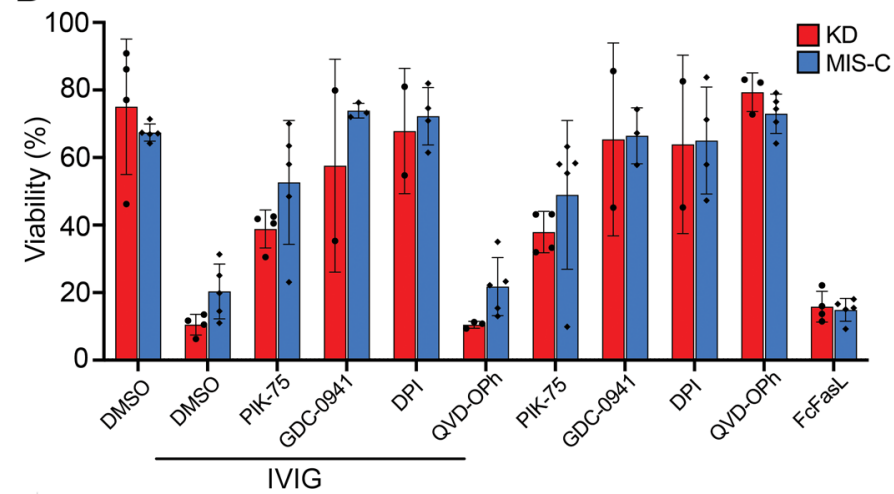

D
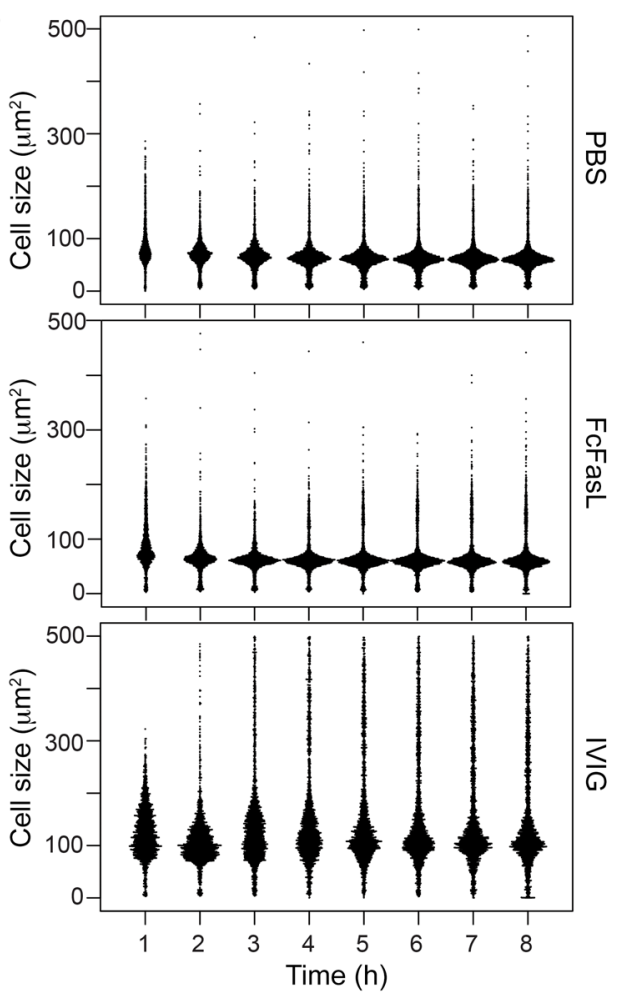

Figure 6. IVIG triggers rapid cell death of neutrophils. (A) Peripheral blood neutrophils undergo unique morphological changes upon exposure to $1 \%$ IVIG. Neutrophils were labeled with $50 \mathrm{nM} \mathrm{CTC} \mathrm{and} \mathrm{incubated} \mathrm{in} \mathrm{the} \mathrm{presence} \mathrm{of} \mathrm{AnnV-AF647} \mathrm{(red)} \mathrm{and} \mathrm{PI} \mathrm{(yellow)} \mathrm{to} \mathrm{track} \mathrm{changes} \mathrm{in} \mathrm{cell} \mathrm{viability.} \mathrm{Representa-}$ tive image from KD neutrophils. Two-hour time point shown. (B) Viability of neutrophils at the 8-hour time point in the presence or absence of $2 \mu M$ PIK75, $10 \mu \mathrm{M}$ GDC-0941, $10 \mu \mathrm{M} \mathrm{DPI}, 10 \mu \mathrm{M}$ QVD-OPh, $100 \mathrm{ng} / \mathrm{mL}$ FcFasL, and 1\% IVIG. (C) Kinetic changes in neutrophil viability classified according to staining with CTC, AnnV, and PI. (D) Changes in cell size of neutrophils treated with $100 \mathrm{ng} / \mathrm{mL}$ FcFasL or $1 \%$ IVIG.

parameters of the population in UMAP plots (Figure 7B). Increases in cell size were observed in IVIG-treated neutrophils isolated from both acute KD and MIS-C patients (Figure 7B), consistent with a nonapoptotic mechanism of action. Neutrophils do not form distinct clusters when undergoing cell death, suggesting that a continuum of states exist, as they undergo a process of cell death in response to stimulation with FasL or IVIG. These data support a unique cell death signaling pathway engaged by IVIG.

\section{Discussion}

Neutrophils have a central role in the pathogenesis of acute KD. Elevated serum G-CSF, neutrophilia, and neutrophil infiltration 

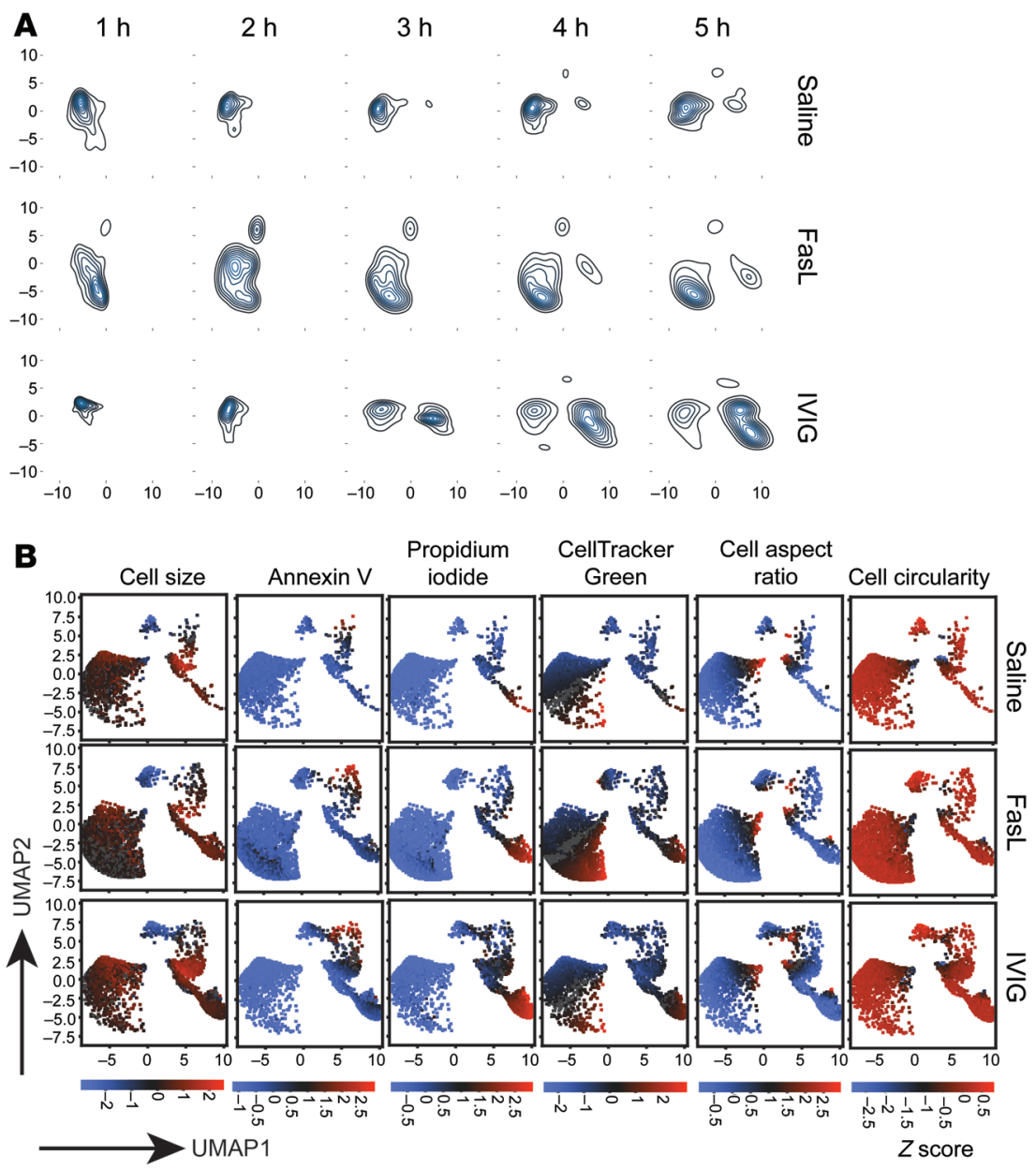

Figure 7. Dimension reduction analysis of neutrophil live-cell imaging reveals a unique IVIC nonapoptotic cell death signature. For each patient, UMAP was performed on morphological and fluorescence parameters from the combined time-course data from neutrophils treated with IVIG, FasL, or saline. (A) A kernel-density estimate plot of cells across wells and over time by UMAP. Contour lines highlight the density of cells. The locations of cells in UMAP plots highlight the trajectories of cells across time and stimuli. The combined UMAP includes cells from all conditions based on equal sampling. (B) Cell features derived from live-cell imaging data overlaid on UMAP plots from cells at time points of 1 to 8 hours. The normalized intensity $z$ score of each feature is shown for each stimuli. of the myocardium and coronary arterial wall are reported features of acute KD (39-41). Progression of KD is associated with a decline in neutrophils and an increase in $\mathrm{CD} 8^{+} \mathrm{T}$ lymphocytes and macrophages in the aneurysm wall, implicating neutrophils in the ensuing adaptive immune response (42). Their role in MIS-C, however, has not been defined, although both neutrophils and NePs are abundant in the circulation in acute cases. In this study, we identified several IL-1 $\beta$-expressing, highly activated neutrophil lineage subsets that disappear from the peripheral circulation following IVIG treatment in both KD and MIS-C patients. We further demonstrated that IVIG can induce the death of neutrophils ex vivo, suggesting that IVIG may in part suppress the acute inflammatory response by reducing the numbers of mature IL-1 $\beta^{+}$ circulating neutrophils. Other factors also clearly contribute to the inflammation in MIS-C, with elevations in IL-1 $\beta$, IL-6, IL-8, IL-10, IL-17, IL-18, and IFN- $\gamma$ and an immune cell phenotypic profile consistent with activation of neutrophils, monocytes, $\mathrm{T}$ cells, and impaired antigen presentation by dendritic cells $(13,20)$. Transient elevation of antibodies reactive with host antigens may also contribute to tissue pathology in MIS-C (20). Further research into disease pathogenesis will help refine our understanding of MIS-C and guide therapeutic choices.

The presence of toxic granulated neutrophils with cytoplasmic swelling, vacuolization, and granulation correlates with the development of coronary artery lesions in KD (43). Recent studies indicate that neutrophils in MIS-C patients also display toxic granulation (44) and are hyperactivated based on CD64 expression $(13,20)$. Whether neutrophils in KD and MIS-C are activated via the same mechanism is unknown. Our CyTOF study on the differential activation marker expression profiles between $\mathrm{KD}$ and MIS-C neutrophils, and indeed other myeloid cells, suggests that these innate immune cells were activated differently or at least at different stages of the disease process. One theory to explain the propensity of these neutrophils to cause damage to the vasculature in KD is a delay in apoptosis of activated neutrophils (41). IVIG is known to induce cell death in human neutrophils. However, the pathways involved remain obscure, and reports of both magnitude of response and kinetics of cell death induction have been inconsistent $(32,35,41)$. IVIG is recognized to exert antiinflammatory effects by diverse mechanisms involving $\mathrm{Fc}$ and $\mathrm{F}\left(\mathrm{ab}^{\prime}\right)_{2}$ components, including IL-10 production by tolerogenic myeloid dendritic cells and regulatory $\mathrm{T}$ cells $(45,46)$. In the case of neutrophils, the in vitro activity of IVIG requires $\mathrm{F}\left(\mathrm{ab}^{\prime}\right){ }_{2}$ fragments $(34,35,47)$. Fc fragments do not replicate or inhibit the actions of IVIG on human neutrophils, suggesting that $\mathrm{F}\left(\mathrm{ab}^{\prime}\right)_{2}$ fragments, or both $\mathrm{Fc}$ and $\mathrm{F}\left(\mathrm{ab}^{\prime}\right)_{2}$, are important in mediating the clinical effects of IVIG on the neutrophil lineage. Our data indicate the presence of a cell death pathway triggered by the $\mathrm{F}\left(\mathrm{ab}^{\prime}\right)_{2}$ component of IVIG, transduced by NADPH oxidase and PI3K, and resulting in the elimination of activated neutrophils and IL- $1 \beta^{+}$neutrophils. 
G-CSF is the dominant regulator of neutrophil production in vivo and is essential for emergency granulopoiesis $(48,49)$. In this study, IVIG treatment was associated with a reduction in plasma G-CSF in patients with KD, likely contributing to reduced neutrophil output from the bone marrow and a loss of IL- $1 \beta^{+}$neutrophils from the circulation. While it is unlikely that G-CSF alone drives the generation and mobilization of pathogenic IL- $1 \beta^{+}$neutrophils in patients with KD or MIS-C, it may be a critical component in the disease process regulated independently of the actions of IVIG on neutrophils described in this study. The presence of circulating IL- $1 \beta^{+}$neutrophils contrasts with IL- $1 \beta$ regulatory mechanisms operating in other innate immune cells. For example, macrophages require a priming step to upregulate pro-IL-1 $\beta$ and inflammasome components for subsequent processing and release (50, 51). Human monocytes can also engage inflammasomes via an alternative activation pathway independently of classical inflammasome stimuli, such as ATP, but do require a signal to upregulate pro-IL-1 $\beta$ (52). In KD and MIS-C patients, the circulating IL-1 $\beta^{+}$ neutrophils appear to be armed effector cells. The high activation status and IL-1 $\beta$ content of these neutrophil lineage cells suggest that they are primed for immediate response and may help initiate or amplify inflammatory responses in organs and tissues. The presence of IL- $1 \beta$ in circulating NePs of patients with KD or MIS-C suggests that these cells may also have immunomodulatory potential like that of mature neutrophils. Host clearance mechanisms and therapeutic strategies that aid in removal of $\mathrm{IL}-1 \beta^{+}$neutrophil lineage cells would therefore be advantageous for a circumscribed inflammatory response. IVIG treatment appears to fulfil this role, eliminating IL- $1 \beta^{+}$neutrophils, likely via cell death. A more detailed time course will be needed to understand the median life span of IL-1 $\beta^{+}$neutrophils following IVIG infusion. These data may also help to explain the effects of IL-1 antagonism for other inflammatory disorders.

Current MIS-C treatment regimens have borrowed heavily from decades of experience with IVIG administration in KD patients. Despite the broad success of IVIG in KD, a subset of children develop aneurysmal coronary arteries, and there is minimal clinical trial data to guide the choice of adjunctive therapy to halt the progression of arterial wall damage and aneurysm formation in these patients. KD is an uncommon disease, so choice of alternative therapies for patients with early aneurysm formation has yet to be guided by adequately powered clinical trials of adjunctive corticosteroids, cyclosporine, and/or inhibitors of TNF and IL-1. Our results are not inconsistent with a role for TNF, but do suggest that, like anakinra, IVIG may also directly impair IL-1 $\beta$ dependent inflammation. Thus, a better understanding of disease pathobiology for both KD and MIS-C will help guide the most effective therapeutic strategies, particularly for those who require additional antiinflammatory therapy beyond IVIG. This study did not address the effects of IVIG on IL-1 $\beta$ processing, nor has it addressed the effects of IVIG on IL- $1 \beta$ expression, processing, and secretion by monocytes and neutrophils in the tissues. Future work will be required to identify the targets of IVIG in neutrophils and to rationally design alternative therapeutic options. A detailed biochemical understanding of pathways engaged by IVIG in different cell types may enable development of recombinant therapies to alleviate demand for IVIG.

\section{Methods}

Study population. All KD patients met the American Heart Association clinical case definition, and all MIS-C patients met the case definition from the Centers for Disease Control and Prevention $(53,54)$. The presence of SARS-CoV-2 antibodies was determined using the Abbott Architect IgG anti-nucleocapsid assay (4 patients). SARS-CoV-2 T cell epitope mapping confirmed the MIS-C diagnosis in 2 patients (24). FCs had fever of at least 3 days duration with at least 1 of the clinical criteria for KD and had one of the following diagnoses: non-SARS-CoV-2 viral syndrome (8), recurrent fever syndrome (1), bacterial lymphadenitis (3), Yersinia enterocolitica bacteremia (1). Research blood samples were collected at the same time as the standard-of-care clinical laboratory testing and, for KD and MIS-C patients, before treatment, 24 hours after IVIG treatment in some subjects, and again, 2 to 6 weeks later after discharge from the hospital.

Flow cytometry. Whole blood leukocytes were stained according to published methods (30). For intracellular IL-1 $\beta$ staining, cells were stained for viability using the LIVE/DEAD Fixable Blue Dead Cell Stain Kit (Thermo Fisher), then fixed and permeabilized using Fixation/Permeabilization Solution (BD Biosciences) and stained with canakinumab, the monoclonal antibody to IL-1 $\beta$, conjugated to Alexa Fluor 647 using an Alexa Fluor 647 Antibody Labeling Kit (Thermo Fisher Scientific). Conjugated IL-1 $\beta$ antibody was added into cell suspension for 30 minutes. Cells were washed twice in $200 \mu \mathrm{L}$ Perm Buffer before acquisition on a LSR Fortessa flow cytometer (BD Biosciences). Data were analyzed using FlowJo (version 10.5).

CyTOF. Metal-conjugated antibodies were purchased directly from Fluidigm for available targets. For all other targets, purified antibodies were purchased and conjugated, and CyTOF was performed following a previously described protocol (30). For viability staining, cells were washed in PBS and stained with cisplatin (Fluidigm) at a final concentration of $5 \mu \mathrm{M}$. RBC-lysed whole blood cells were incubated with metal-conjugated CD16, CD32, and CD64 for 30 minutes at $4^{\circ} \mathrm{C}$ to block $\mathrm{Fc}$ receptors prior to surface staining for 30 minutes at $4^{\circ} \mathrm{C}$. After surface staining, cells were washed with staining buffer and fixed with $1.6 \%$ paraformaldehyde (PFA) (Thermo Fisher) for 15 minutes at room temperature. After removal of the $1.6 \%$ PFA, cells were washed with $1 \times$ Perm buffer and resuspended in $1 \times$ Fixation/ Permeabilization solution (BD Biosciences) for incubation overnight at $4^{\circ} \mathrm{C}$. Cells were then washed with $1 \times$ Perm buffer and stained with metal-conjugated canakinumab for 30 minutes at $4^{\circ} \mathrm{C}$. After the intracellular staining, cells were washed with $1 \times$ Perm buffer and resuspended in $1 \mathrm{~mL}$ of $125 \mathrm{nM}$ intercalation solution (Fluidigm), followed by incubation overnight at $4^{\circ} \mathrm{C}$. Before acquisition, cells were washed twice with $1 \times$ Perm buffer and resuspended in CAS with a 1:10 dilution of EQ Four Element Calibration beads (Fluidigm) and filtered through a $35 \mu \mathrm{m}$ nylon mesh filter cap (Corning, Falcon). Samples were analyzed on a Helios 2 CyTOF Mass Cytometer (Fluidigm) at an event rate less than or equal to 500 events per second. CyTOF data files were normalized using the bead-based Normalizer 21 and analyzed on the OMIQ data science platform.

G-CSF Meso Scale. G-CSF was assessed using a Meso Scale S-PLEX Human G-CSF Kit (MSD) from plasma samples of 5 patients with acute KD (illness days 3-10) and again after IVIG (illness days 19-86).

RNA expression. RNA was extracted using RNeasy kits (QIAGEN) and reverse transcribed using the PrimeScript RT Reagent Kit (Takara). cDNA was analyzed by IL1A and IL1B TaqMan quantitative PCR (qPCR) 


\section{Table 2. Antibodies}

\begin{tabular}{|c|c|c|}
\hline Reagent or resource & Source & Catalog no. \\
\hline \multicolumn{3}{|l|}{ CyTOF antibodies } \\
\hline Anti-human CD45 (HI30)-89Y & Fluidigm & $3089003 B$ \\
\hline Anti-human CD4 (RPA-T4)-145Nd & Fluidigm & $3145001 B$ \\
\hline Anti-human CD56 (R19-760)-176Yb-100 tests & Fluidigm & 3176013B \\
\hline Anti-human CD19 (HIB19)-165Ho & Fluidigm & $3165025 B$ \\
\hline Anti-human HLA-DR (L243)-174Yb & Fluidigm & $3174001 B$ \\
\hline Anti-human CD33 (WM53)-169Tm & Fluidigm & $3169010 \mathrm{~B}$ \\
\hline Anti-Mouse CD11b (M1/70)-148Nd & Fluidigm & 3148003B \\
\hline Anti-human CD66b (80H3)-152Sm & Fluidigm & $3152011 \mathrm{~B}$ \\
\hline Anti-human CD32 (FUN-2)-153Eu & Fluidigm & 3153018B \\
\hline Anti-human CD16 (3G8)-209Bi & Fluidigm & $3209002 B$ \\
\hline Anti-human CD117/c-kit (104D2)-143Nd & Fluidigm & $3143001 B$ \\
\hline Anti-human CD34 (581)-166Er & Fluidigm & $3166012 B$ \\
\hline Anti-human CD49d (9F10)-141Pr & Fluidigm & $3141004 \mathrm{~B}$ \\
\hline Anti-human CD101 (BB27)-158Gd & Fluidigm & $3158020 B$ \\
\hline Anti-human CD15/SSEA-1 (W6D3)-172Yb & Fluidigm & $3172021 \mathrm{~B}$ \\
\hline Anti-human CD10 (HI10a)-156Gd & Fluidigm & $3156001 B$ \\
\hline Anti-human CD79B (CB3-1)-162Dy & Fluidigm & $3162008 B$ \\
\hline Anti-human CD71 (OKT-9)-168Er & Fluidigm & $3168014 \mathrm{~B}$ \\
\hline Anti-human CD123/LL-3R (6H6)-151Eu & Fluidigm & $3151001 \mathrm{~B}$ \\
\hline Anti-human CD11a (HI111)-142Nd & Fluidigm & $3142006 B$ \\
\hline Anti-human CD162 (KPL-1)-161Dy & Fluidigm & $3161026 B$ \\
\hline Anti-human CD54 (HA58)-170Er & Fluidigm & $3170014 \mathrm{~B}$ \\
\hline Anti-human CD63 (H5C6)-150Nd & Fluidigm & $3150021 \mathrm{~B}$ \\
\hline Anti-human/mouse CD49F (CoH3)-164Dy & Fluidigm & $3164006 B$ \\
\hline Anti-human CD182/CXCR2 (5E8/CXCR2)-147Sm & Fluidigm & $3147010 \mathrm{~B}$ \\
\hline Anti-human CD184/CXCR4 (12G5)-175Lu & Fluidigm & $3175001 B$ \\
\hline Cell ID 127 IdU & Fluidigm & 201127 \\
\hline Purified anti-human CD3 (Maxpar Ready) antibody & BioLegend & 300443 \\
\hline Purified anti-human CD41 (Maxpar Ready) antibody & BioLegend & 303721 \\
\hline Purified anti-human CD235ab (Maxpar Ready) antibody & BioLegend & 306615 \\
\hline Purified anti-human CD64 (Maxpar Ready) antibody & BioLegend & 305029 \\
\hline Purified anti-human CD86 (Maxpar Ready) antibody & BioLegend & 305435 \\
\hline Purified anti-human CD14 (Maxpar Ready) antibody & BioLegend & 301843 \\
\hline Purified anti-human CD11c (Maxpar Ready) antibody & BioLegend & 301639 \\
\hline Purified anti-human CD203c (E-NPP3) antibody & BioLegend & 324602 \\
\hline Purified anti-human Siglec- 8 antibody & BioLegend & 347102 \\
\hline Purified anti-human CD62L (Maxpar Ready) antibody & BioLegend & 304835 \\
\hline Purified anti-mouse/human CD44 (Maxpar Ready) antibody & BioLegend & 103051 \\
\hline Purified anti-human CD95 (Fas) (Maxpar Ready) antibody & BioLegend & 305631 \\
\hline Purified anti-human CD178 (Fas-L) antibody & BioLegend & 306402 \\
\hline \multicolumn{3}{|l|}{ Flow cytometry antibodies } \\
\hline Brilliant Violet 570 anti-human CD45 antibody & BioLegend & 304034 \\
\hline PE/Dazzle 594 anti-human Siglec-8 antibody & BioLegend & 347110 \\
\hline PE/Dazzle 594 anti-human CD203c (E-NPP3) antibody & BioLegend & 324624 \\
\hline PerCP/Cyanine5.5 anti-human CD3 antibody & BioLegend & 300328 \\
\hline PerCP/Cyanine5.5 anti-human CD19 antibody & BioLegend & 363016 \\
\hline PerCP/Cyanine5.5 anti-human CD56 (NCAM) antibody & BioLegend & 304626 \\
\hline Brilliant Violet 605 anti-human CD86 antibody & BioLegend & 305430 \\
\hline APC/Cyanine7 anti-human HLA-DR antibody & BioLegend & 307618 \\
\hline PE anti-human CD66b antibody & BioLegend & 305106 \\
\hline APC-R700 mouse anti-human CD117 & BD Biosciences & 565195 \\
\hline BV421 mouse anti-human CD34 & BD Biosciences & 562577 \\
\hline Brilliant Violet 510 anti-human CD10 antibody & BioLegend & 312220 \\
\hline Brilliant Violet 785 anti-human CD16 antibody & BioLegend & 302046 \\
\hline
\end{tabular}

with reference gene TBP following the manufacturer's instruction (TaqMan primers: IL1A Hs00174092_m1, IL1B Hs01555410_m1, TBP Hs00427620_m1; Life Technologies).

Live-cell imaging. Neutrophils were isolated according to published methods (55). Neutrophils were labeled with 50 nM CellTracker Green (Life Technologies) for 15 minutes at $37^{\circ} \mathrm{C}$ in serum-free DMEM, and $1 \times 10^{5}$ neutrophils were plated in 96-well optical bottom plates (Corning, 3904) in $100 \mathrm{ng} / \mathrm{mL}$ recombinant human G-CSF (Amgen). Neutrophil viability was monitored using AnnV-Alexa Fluor 647 (BioLegend) to detect phosphatidylserine and $0.5 \mu \mathrm{g} / \mathrm{mL}$ PI (Sigma-Aldrich). Cells were treated with $1 \%$ IVIG (10\% v/v from GAMMAGARD LIQUID 10\%), 50 $\mu \mathrm{g} / \mathrm{mL}$ Fc (Life Meridian Science), $10 \mu \mathrm{M}$ PIK-75 (Cayman Chemical), $10 \mu \mathrm{M}$ GDC-0941 (Cayman Chemical), $10 \mu \mathrm{M}$ diphenyleneiodonium chloride (Cayman Chemical), $10 \mu \mathrm{M}$ zVD-fmk (Cayman Chemical), $10 \mu \mathrm{M}$ QVD-OPh (Cayman Chemical), $10 \mu \mathrm{M}$ necrostatin-2 (Cayman Chemical), 300 ng/mL FcFasL (Adipogen), $1 \mu \mathrm{M}$ liproxstatin-1, (Cayman Chemicals), $1 \mu \mathrm{M}$ ferrostatin-1 (Cayman Chemical), $1 \mu \mathrm{M}$ BBCl-amidine (Cayman Chemical), $1 \mu \mathrm{M}$ GSK199 (Cayman Chemical), $1 \mu \mathrm{M}$ GSK484 (Cayman Chemical), and 10 $\mu \mathrm{M}$ MitoTEMPO (Cayman Chemical). Images from triplicate fields of view were captured with a $10 \times 0.45$ NA objective every hour using a Nikon Ti2-E microscope with a Qi-2 camera and Nikon Elements 5.02.02 acquisition software utilizing the Jobs module. Cells were maintained at $37^{\circ} \mathrm{C}$ $+5 \% \mathrm{CO}_{2}$ using an Okolab $\mathrm{H} 301$ Bold Line stage top incubator. Three nonoverlapping $3.125 \mathrm{~mm}^{2}$ zones within each well were imaged for red, green, and far-red fluorescence using a SpectraX light engine (Lumencor) with individual LFOV filter cubes (Semrock) (excitation/emission maxima at $554 / 609,470 / 525$, and $618 / 698 \mathrm{~nm}$, respectively).

Cell death kinetics were analyzed using a custom macro in the Fiji distribution of Image (NIH) $(56,57)$. The macro processed each time point folder that was generated by the Nikon Ti2 imaging system, and time image stacks were constructed of each well field position. The macro segmented each cell population in each frame of the time stacks using custom-scripted code for the Fiji distribution of Image J and the deep learning plugin StarDist, and then population percentages were calculated for each time point from the extracted binary images (36). Using these masks, each cell population was defined using Boolean arithmetic. The logic was constructed so that cells were only counted once. Data were logged and percentage populations were calculated at each time point.

DeathTrackR data visualization. ImageJ data logs from live-cell imaging analyses were visualized using the R Shiny app DeathTrackR (https://croker.shinyapps.io/ project/) and the $\mathrm{R}$ Shiny app CellSizeTrackR (https:// croker.shinyapps.io/cellsizetrackr/). The DeathTrackR package was developed using Shiny in $\mathrm{R}$ 3.6.3., the $\mathrm{R}$ packages Tidyverse and plotrix for basic data loading and plotting utilities, and shinydashboard for module management (58-61). CellSizeTrackR requires $\mathrm{R}$ packages dplyr and beeswarm for basic data handling and plotting 
utilities (62). The code for DeathTrackR is available online (commit ID: 5d87dd366ae856caedb1af638f50b1f1c6bd8283; commit URL: https://github.com/DeathTrackR/DeathTrackR/commits/ master). The code for CellsizeTrackR is available online (commit ID: 78f33de92492e425cba68fod6bfa687f67841438; commit URL: https://github.com/WeiqiPeng0/CellSizeTrackR/commits/main).

UMAP analysis of live-cell imaging data. Intensities of AnnV, CTG, and PI were extracted for single cells, along with cell size, cell circularity, and cell aspect ratio, for a total of 6 features. The $\log _{10}$ value was calculated for each feature, which was then $z$ scored. Cell debris was removed by applying a gate on objects less than $0.5829 \mu \mathrm{m}^{2}$ in size $(z$ score of -3 ). Other outlier features with a $z$ score of more or less than 3 were additionally removed. The UMAP algorithm was implemented using the UMAP package (version 0.3.10) in Python (38). We subsampled $25 \%$ of the cell population and extracted $2 \mathrm{D}$ embeddings for each cell along with the feature weights corresponding to the contribution of each feature to each dimension. This was done on 3 different random subsamples, and all produced similar UMAP distributions. To control global patterns and cluster separation, we set n_neighbors to 200 (default 15), which controls the size of the local neighborhood for each UMAP point, and min_dist to 0 (default 0.1), which controls how tightly the points are allowed to be packed together. Hexbins were generated using matplotlib (version 3.1.1). Plotting and analysis were done in Python 3.6. For the hexbin plots, the median value of the attribute was taken within each bin.

Code availability. The custom-scripted macro used for automated image analysis of live-cell imaging data is available online (https:// figshare.com/s/115585435648ec5d974c).

Statistics. Statistical analyses were performed with Prism software (GraphPad). For Figures 2 and 3, differences between groups were determined via 1-way ANOVA and Tukey's multiple-comparison tests with a single pooled variance. For Figures 4 and 5, differences between pre- and post-IVIG groups were determined via 2-tailed, unpaired $t$ tests. For Figure 5G, differences between before and after IVIG treatment were determined via paired $t$ tests. Lines on dot plots indicate median with IQR. A $P$ value of less than 0.05 was considered significant.
Study approval. Patients were enrolled in a study approved by the Institutional Review Board of UCSD (no. 140220), and parental signed consent and child assent were obtained as appropriate.

\section{Author contributions}

YPZ, IS, JTK, NEL, AHT, JCB, BAC, LB, and HMH conceived and designed the study. YPZ, IS, EG, SA, NEL, JTK, AHT, JCB, and BAC analyzed the data. AHT, JCB, BAC, HMH, and NEL provided funding. YPZ, IS, JCL, EM, CS, JTK, AHT, JCB, BAC, AF, LB, and $\mathrm{HMH}$ performed experiments. IS, CJN, WP, SGL, LNNL, YL, HM, $\mathrm{HX}$, and CJP were involved in development of software and code for data analysis and visualization. AHT, NEL, JTK, JCB, BAC, and $\mathrm{HMH}$ helped oversee the project and supervised personnel involved with the project. YPZ, IS, JTK, NEL, AHT, JCB, BAC, LB, and $\mathrm{HMH}$ wrote the manuscript.

\section{Acknowledgments}

This work was supported by R61HD105590 (to JCB), the Patient Centered Outcomes Research Institute (CER-1602-3447 to JCB), NIH grant RO1HL124209 (to BAC), NIH grant RO1HL140898$03 \mathrm{~S} 1$ (to JCB, AHT, and HMH), the American Asthma Foundation (BAC), the American Heart Association (Career Development Award to YPZ), the Novo Nordisk Foundation provided to the Technical University of Denmark (NNF20SA0066621 to NEL), the National Institute of General Medical Sciences (R35 GM119850 to NEL), and the American Academy of Allergy, Asthma and Immunology Foundation (to LB). This work was supported by the Shared Instrumentation Grant Program (S10 OD018499 to the Flow Cytometry Core Facility at the La Jolla Institute) See Supplemental Acknowledgments for details on the Pediatric Emergency Medicine Kawasaki Disease Research Group Consortium.

Address correspondence to: Ben A. Croker or Jane C. Burns, Department of Pediatrics, School of Medicine, UC San Diego, 9500 Gilman Drive MC 0760, La Jolla, California 92093-0760, USA. Email: bcroker@health.ucsd.edu (BAC); Email: jcburns@ health.ucsd.edu (JCB).
1. Jiang L, et al. COVID-19 and multisystem inflammatory syndrome in children and adolescents. Lancet Infect Dis. 2020;20(11):e276-e288.

2. Feldstein LR, et al. Multisystem inflammatory syndrome in U.S. Children and adolescents. N Engl JMed. 2020;383(4):334-346.

3. Cheung EW, et al. Multisystem inflammatory syndrome related to COVID-19 in previously healthy children and adolescents in New York City. JAMA. 2020;324(3):294-296.

4. Riphagen S, et al. Hyperinflammatory shock in children during COVID-19 pandemic. Lancet. 2020;395(10237):1607-1608.

5. Toubiana J, et al. Kawasaki-like multisystem inflammatory syndrome in children during the covid-19 pandemic in Paris, France: prospective observational study. BMJ. 2020;369:m2094.

6. Verdoni L, et al. An outbreak of severe Kawasakilike disease at the Italian epicentre of the SARSCoV-2 epidemic: an observational cohort study. Lancet. 2020;6736(10239):1-1778.

7. Whittaker E, et al. Clinical characteristics of 58 children with a pediatric inflammatory multisystem syndrome temporally associated with SARS CoV-2. JAMA. 2020;324(3):259-269.

8. Tanaka N. Pathological study of Kawasaki disease (MCLS): with special reference to sequelae. Jpn J Med Sci Biol. 1979;32(4):245-246.

9. Yonesaka S, et al. Endomyocardial biopsy in children with Kawasaki disease. Acta Paediatr Jpn. 1989;31(6):706-711.

10. Gordon JB, et al. The spectrum of cardiovascular lesions requiring intervention in adults after Kawasaki disease. JACC Cardiovasc Interv. 2016;9(7):687-696.

11. Gordon JB, et al. When children with Kawasaki disease grow up: Myocardial and vascular complications in adulthood. J Am Coll Cardiol. 2009;54(21):1911-1920.

12. Kato H, et al. Long-term consequences of Kawasaki disease. A 10- to 21-year follow-up study of 594 patients. Circulation. 1996;94(6):1379-1385.

13. Carter MJ, et al. Peripheral immunophenotypes in children with multisystem inflammatory syn- drome associated with SARS-CoV-2 infection. Nat Med. 2020;26(11):1701-1707.

14. Alsaied T, et al. Review of cardiac involvement in multisystem inflammatory syndrome in children. Circulation. 2020;143(1):78-88.

15. Ahmed M, et al. Multisystem inflammatory syndrome in children: a systematic review. EClinicalMedicine. 2020;26:100527.

16. Burns JC, Franco A. The immunomodulatory effects of intravenous immunoglobulin therapy in Kawasaki disease. Expert Rev Clin Immunol. 2015;11(7):819-825.

17. Henderson LA, et al. American College of Rheumatology Clinical guidance for multisystem inflammatory syndrome in children associated with SARS-CoV-2 and hyperinflammation in pediatric COVID-19: version 2. Arthritis Rheumatol. 2021;73(4):e13-e29.

18. Zuo Y, et al. Neutrophil extracellular traps in COVID-19. JCI Insight. 2020;5(11):e138999.

19. Barnes BJ, et al. Targeting potential drivers of COVID-19: neutrophil extracellular traps. JExp 
Med. 2020;217(6):e20200652.

20. Gruber C, et al. Mapping systemic inflammation and antibody responses in multisystem inflammatory syndrome in children (MIS-C). Cell. 2020;183(4):982-995.

21. Hoang LT, et al. Global gene expression profiling identifies new therapeutic targets in acute Kawasaki disease. Genome Med. 2014;6(11):541.

22. Wakita D, et al. Role of interleukin-1 signaling in a mouse model of Kawasaki disease-associated abdominal aortic aneurysm. Arterioscler Thromb Vasc Biol. 2016;36(5):886-897.

23. Lehman TJ, Mahnovski V. Animal models of vasculitis. Lessons we can learn to improve our understanding of Kawasaki disease. Rheum Dis Clin North Am. 1988;14(2):479-487.

24. Grifoni A, et al. Targets of $T$ cell responses to SARS-CoV-2 Coronavirus in humans with COVID-19 disease and unexposed individuals. Cell. 2020;181(7):1489-1501.

25. Huet T, et al. Anakinra for severe forms of COVID-19: a cohort study. Lancet Rheumatol. 2020;2(7):e393-e400.

26. Fury W, et al. Transcript abundance patterns in Kawasaki disease patients with intravenous immunoglobulin resistance. Hum Immunol. 2010;71(9):865-873.

27. Tremoulet AH, et al. Rationale and study design for a phase I/IIa trial of anakinra in children with Kawasaki disease and early coronary artery abnormalities (the ANAKID trial). Contemp Clin Trials. 2016;48:70-75.

28. Rondeau JM, et al. The molecular mode of action and species specificity of canakinumab, a human monoclonal antibody neutralizing IL-1 $\beta$. MAbs. 2015;7(6):1151-1160.

29. Dinh HQ, et al. Coexpression of CD71 and CD117 identifies an early unipotent neutrophil progenitor population in human bone marrow. Immunity. 2020;53(2):319-334.

30. Zhu YP, et al. Identification of an early unipotent neutrophil progenitor with pro-tumoral activity in mouse and human bone marrow. Cell Rep. 2018;24(9):2329-2341.

31. Evrard M, et al. Developmental analysis of bone marrow neutrophils reveals populations specialized in expansion, trafficking, and effector functions. Immunity. 2018;48(2):364-379.

32. Tsujimoto $\mathrm{H}$, et al. Intravenous immunoglobulin therapy induces neutrophil apoptosis in Kawasaki disease. Clin Immunol. 2002;103(2):161-168.

33. Graeter S, et al. Granulocyte death mediated by specific antibodies in intravenous immunoglobulin (IVIG). Pharmacol Res. 2020;154:104168.

34. von Gunten S, et al. Immunologic and functional evidence for anti-Siglec-9 autoantibodies in intravenous immunoglobulin preparations. Blood. 2006;108(13):4255-4259.

35. Schneider C, et al. IVIG regulates the survival of human but not mouse neutrophils. Sci Rep. 2017;7(1):1296.

36. Schmidt $\mathrm{U}$, et al. Cell detection with star-convex polygons. Paper presented at: Medical Image Computing and Computer Assisted Intervention - MICCAI 2018; September 16-20, 2018; Grenada, Spain. https://doi.org/10.1007/978-3-03000934-2_30. Accessed August 30, 2021.

37. von Gunten S, et al. Immunologic and functional evidence for anti-Siglec- 9 autoantibodies in intravenous immunoglobulin preparations. Blood. 2006;108(13):4255-4259.

38. McInnes L, Healy J. UMAP:Uniform Manifold Approximation and Projection for dimension reduction [preprint]. https://arxiv.org/ $\mathrm{abs} / 1802.03426 \mathrm{v} 3$. Posted on arXiv September $18,2020$.

39. Andreozzi L, et al. A master role for neutrophils in Kawasaki syndrome. Immunol Lett. 2017;184:112-114.

40. Suzuki H, et al. Serum levels of neutrophil activation cytokines in Kawasaki disease. Pediatr Int . 2001;43(2):115-119.

41. Tsujimoto H, et al. Delayed apoptosis of circulating neutrophils in Kawasaki disease. Clin Exp Immunol. 2001;126(2):355-364.

42. Brown TJ, et al. CD8 T lymphocytes and macrophages infiltrate coronary artery aneurysms in acute Kawasaki disease. J Infect Dis. 2001;184(7):940-943.

43. Takeshita S, et al. Studies of peripheral blood toxic neutrophils as a predictor of coronary risk in Kawasaki disease - the pathogenetic role of hematopoietic colony-stimulating factors (GM-CSF, G-CSF). Acta Paediatr Jpn. 1990;32(5):508-514.

44. Diorio C, et al. Multisystem inflammatory syndrome in children and COVID-19 are distinct presentations of SARS-CoV-2. J Clin Invest. 2020;130(11):5967-5975.

45. Franco A, et al. Pediatric tolerogenic DCs expressing CD4 and immunoglobulin-like transcript receptor (ILT)- 4 secrete IL-10 in response to $\mathrm{Fc}$ and adenosine. Eur JImmunol. 2018;48(3):482-491.

46. Franco A, et al. Specificity of regulatory $\mathrm{T}$ cells that modulate vascular inflammation. Autoim munity. 2014;47(2):95-104.

47. Graeter S, et al. Granulocyte death mediated by specific antibodies in intravenous immunoglobulin (IVIG). Pharmacol Res. 2020;154:104168.

48. Lieschke GJ, et al. Mice lacking granulocyte colonystimulating factor have chronic neutropenia, granulocyte and macrophage progenitor cell deficiency, and impaired neutrophil mobilization. Blood.1994;84(6):1737-1746.

49. Liu F, et al. Interleukin- 6 and the granulocyte colony-stimulating factor receptor are major independent regulators of granulopoiesis in vivo but are not required for lineage commitment or terminal differentiation. Blood.1997;90(7):2583-2590.

50. Martinon F, et al. Gout-associated uric acid crystals activate the NALP3 inflammasome. Nature. 2006;440(7081):237-241.

51. Martinon F, et al. The inflammasome: a molecular platform triggering activation of inflammatory caspases and processing of prolL-beta. $\mathrm{Mol}$ Cell. 2002;10(2):417-426.

52. Gaidt MM, et al. Human monocytes engage an alternative inflammasome pathway. Immunity. 2016;44(4):833-846.

53. McCrindle BW, et al. Diagnosis, treatment, and long-term management of Kawasaki disease: a scientific statement for health professionals from the American Heart Association. Circulation. 2017;135(17):e927-e999.

54. Centers for Disease Control and Prevention. Multisystem Inflammatory Syndrome in Children (MIS-C) Associated with Coronavirus Disease 2019 (COVID-19). https://emergency.cdc. gov/han/2020/han00432.asp. Updated May 14, 2020. Accessed August 30, 2021.

55. D'Cruz AA, et al. The pseudokinase MLKL activates PAD4-dependent NET formation in necroptotic neutrophils. Sci Signal. 2018;11(546):eaao1716.

56. Speir M, et al. Ptpn6 inhibits caspase-8- and Ripk3/Mlkl-dependent inflammation. Nat Immunol. 2020;21(1):54-64.

57. Schindelin J, et al. Fiji: an open-source platform for biological-image analysis. Nat Methods. 2012;9(7):676-682.

58. shiny: Web Application Framework for R. Version 1.6.0. Chang W, et al.; 2021. Accessed August 30, 2021. https://cran.r-project.org/web/packages/ shiny/index.html.

59. $R$ : A language and environment for statistical computing. Version 4.1.1. R Foundation; 2021. Accessed August 30, 2021. https://www.r-project.org/.

60. dplyr: A Grammar of Data Manipulation. Version 1.0.7. Wickham H, et al.; 2021. Accessed August 30, 2021. https://CRAN.R-project.org/ package $=$ dplyr.

61. Lemon J. Plotrix: a package in the red light district of R. R-News. 2006;6:8-12.

62. beeswarm: The Bee Swarm Plot, an Alternative to Stripchart. Version 0.4.0. Eklund A, Trimble J; 2021. Accessed August 30, 2021. https:// CRAN.R-project.org/package=beeswarm. 